(Supporting Information)

Preparation and biological evaluation of ${ }^{10} \mathrm{~B}$-enriched 3-[5-\{2-(2,3-dihydroxyprop-1yl)-o-carboran-1-yl\}pentan-1-yl]thymidine (N5-2OH): A new boron delivery agent for boron neutron capture therapy of brain tumors

Youngjoo Byun, ${ }^{\dagger} *$ B. T. S, Thirumamagal, ${ }^{\dagger}$ Weilian Yang, ${ }^{\S}$ Staffan Eriksson, ${ }^{\ddagger}$ Rolf F. Barth, ${ }^{\S}$ and Werner Tjarks ${ }^{\dagger}$

${ }^{\dagger}$ Division of Medicinal Chemistry \& Pharmacognosy in the College of Pharmacy and ${ }^{\S}$ Department of Pathology, The Ohio State University, Columbus, Ohio 43210, USA, ${ }^{\ddagger}$ Department of Molecular Biosciences, Division of Veterinary Medical Biochemistry, Swedish University of Agricultural Sciences, The Biomedical Center, Uppsala, SE-75123 Uppsala, Sweden.

Contents:

1. General (S1)

2. ESI-Mass Spectra (S2)

3. NMR spectra of 1A (page S3-S6)

4. Scheme 1. Synthesis of compound 11, Experimental section (S7-S10)

5. Scheme 2. Synthesis of compound 12, Experimental section (S11-S14)

6. Partial ${ }^{1} \mathrm{H}-\mathrm{NMR}$ spectra of 10A, 10B, 10C, and 1A with $(S)-(+)-2,2,2$-trifluoro-1- (9anthryl)-ethanol in $\mathrm{CDCl}_{3}$ at $300 \mathrm{~K}$ (S15)

7. HPLC spectrum of a mixture of 10C, 11 and 12 (S16)

8. HPLC purity spectra of all target compounds using reversed-phase C-18. Mobile phases: Acetonitrile and water, methanol and water (S17-S23)

9. Calculated Energy, RMS gradient, and dipole moment of the minimized structures for 10A, (R)-epimers of 11 and 12 at B3LYP/6-31G* level using Gaussian 03 program. program (S24)

10. Human TK1 homology model docked with the (R)-epimer of 11 (S25)

1. General

${ }^{1} \mathrm{H}-\mathrm{NMR},{ }^{13} \mathrm{C}-\mathrm{NMR}$, and ${ }^{11} \mathrm{~B}-\mathrm{NMR}$ spectra were obtained on Bruker 250,300 or 400 MHz FT-NMR instruments. Chemical shifts are reported in parts per million (ppm). The coupling constants are reported in Hertz $(\mathrm{Hz})$. High resolution electrospray ionization (HR-ESI) mass spectra were recorded on a Micromass QTOF-Electrospray mass spectrometer and a 3-Tesla Finnigan FTMS-2000 Fourier Transform mass spectrometer at The Ohio State University Campus Chemical Instrumentation Center (OSU-CCIC) and Analytical HPLC data of the target compounds were obtained with reversed phase C18 (RP-18) LiChrosphere $100 \AA$ [5 $\mu \mathrm{m}$ ] columns (Merck,) using an Rainin HPLC instrument equipped with a Dynamax DA controller, HPXL pumps, and a Dynamax UV-1 detector (Rainin Instrument Company Inc., Woburn, MA, USA). HPLC grade water, methanol, and acetonitrile were used as solvents. A water/methanol gradient (100:0 to 30:70 over 10 $\mathrm{min}$, from $30: 70$ to $10: 90$ over $20 \mathrm{~min}$, and from 10:90 to 0:100 over $10 \mathrm{~min}$ ) with a flow rate of $1 \mathrm{~mL} / \mathrm{min}$ was applied for all target compounds. A water/acetonitrile gradient (100:0 to $70: 30$ over $5 \mathrm{~min}$, from 70:30 to 40:60 over $25 \mathrm{~min}$, from 40:60 to 0:100 over $20 \mathrm{~min})$. 


\section{ESI-Mass Spectra}

High-resolution mass spectrum of $\mathbf{1 A}$

LCT electrospray $\quad$ (614) 292-4821

Lo82405d 130 (2.383) AM (Cen, 4, 80.00, Ht,5000.0,0.00,1.00); Sm (SG, 2×3.00); Cm (129:137)

100

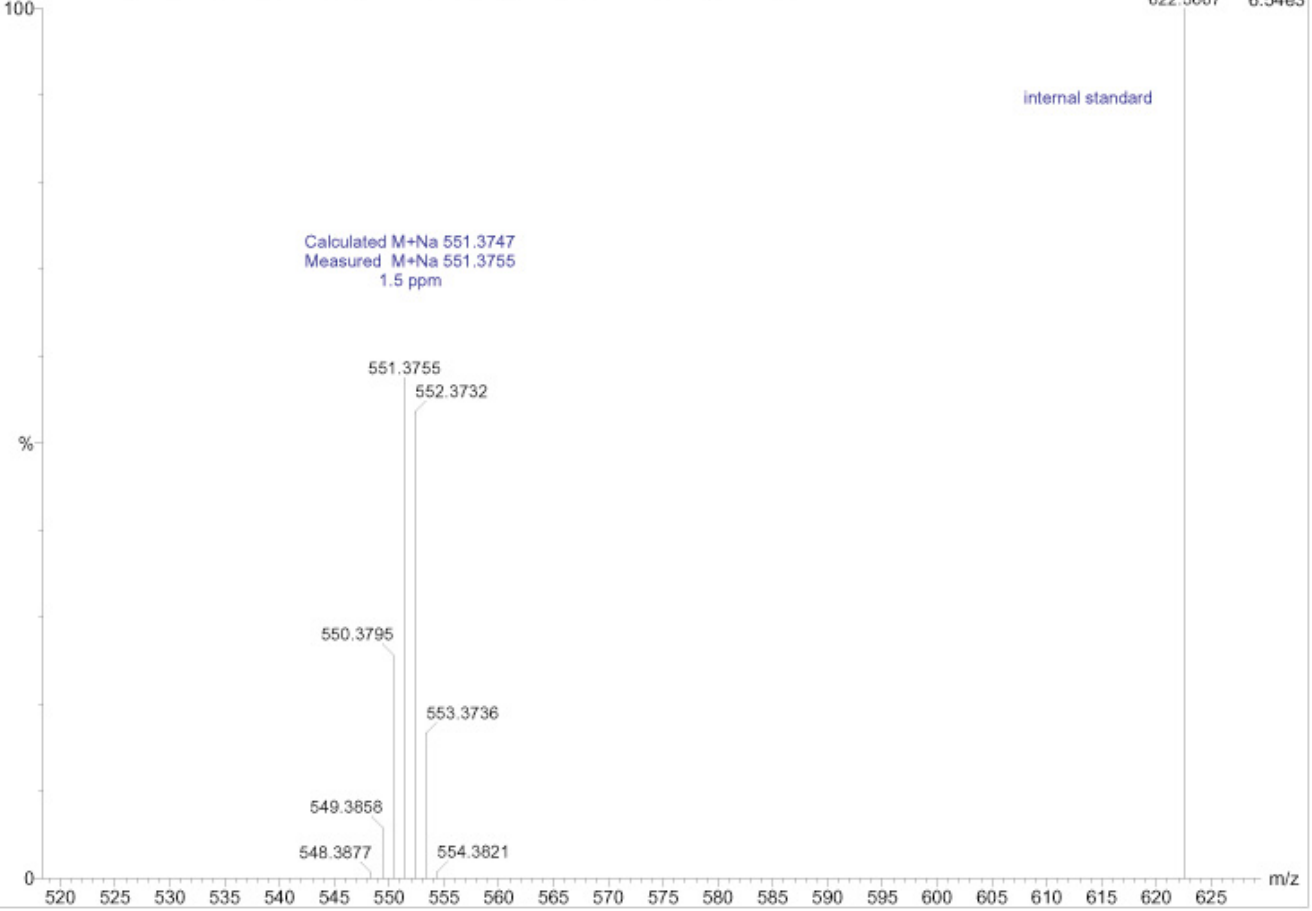

\section{High-resolution mass spectrum of $\mathbf{1 B}$}

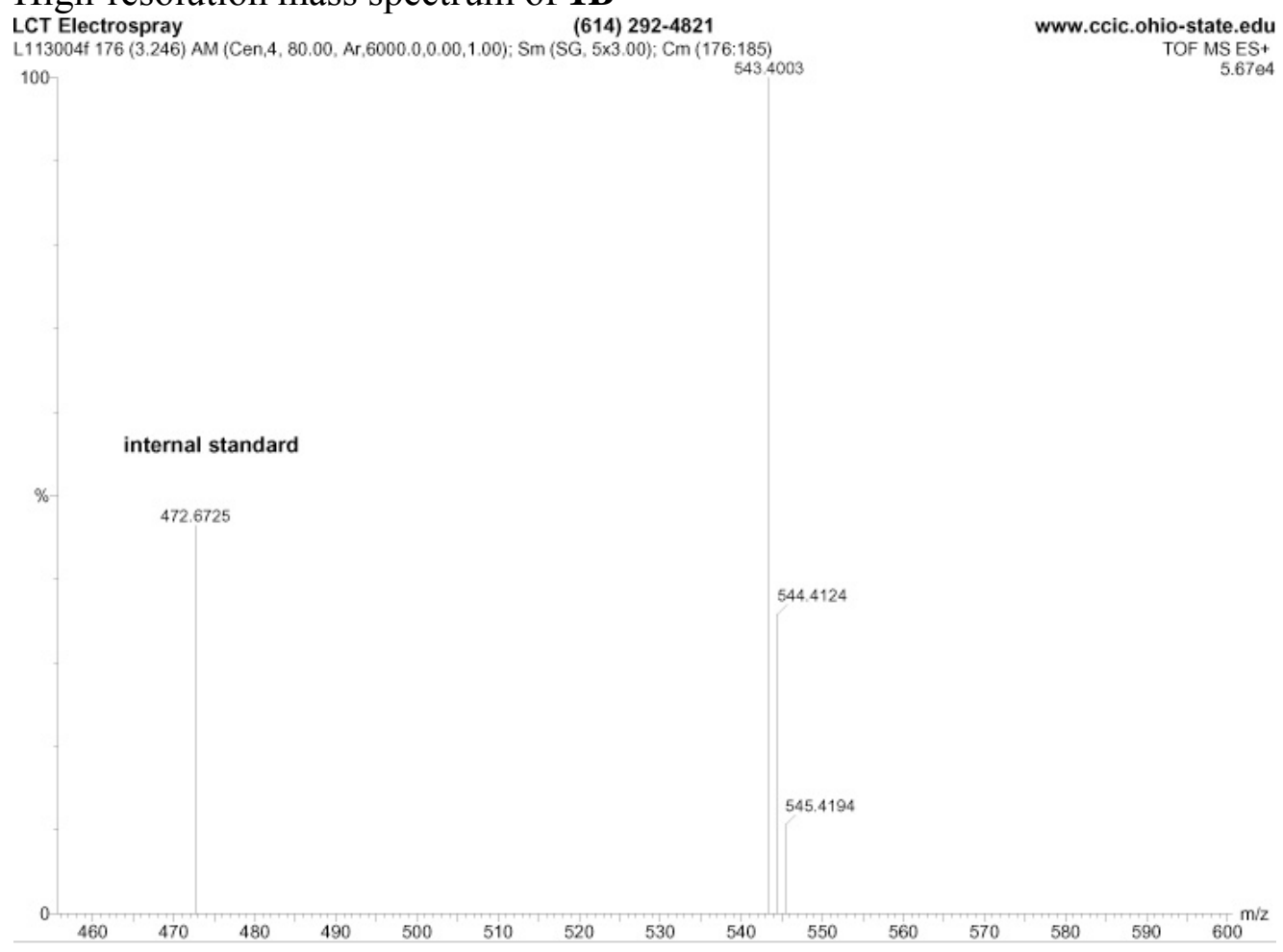


3. NMR spectra of $\mathbf{1 A}$

2D-HMBC spectrum of $\mathbf{1 A}$

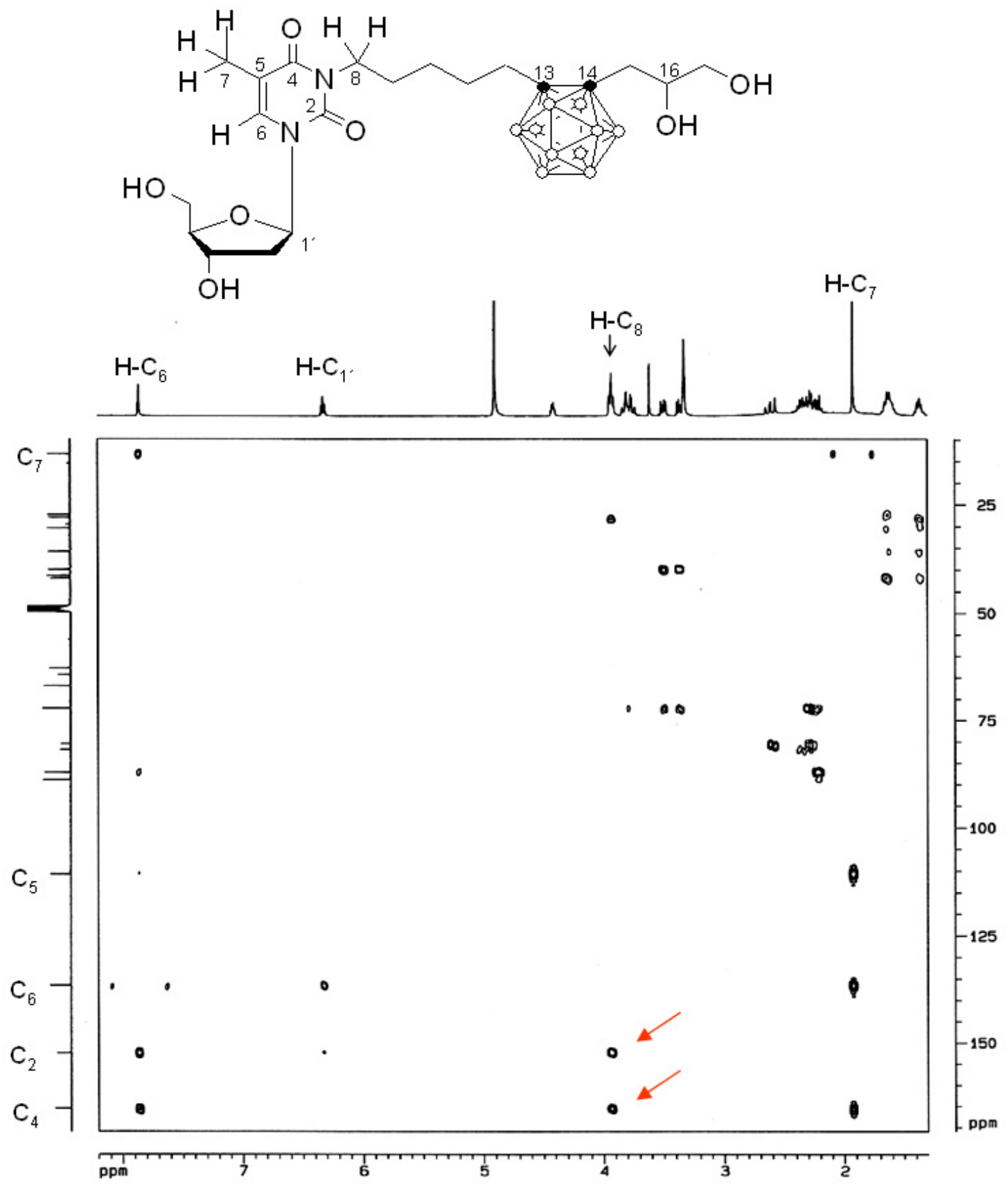


${ }^{1} \mathrm{H}-\mathrm{NMR}$ and 2D-COSY spectra of $\mathbf{1 A}$
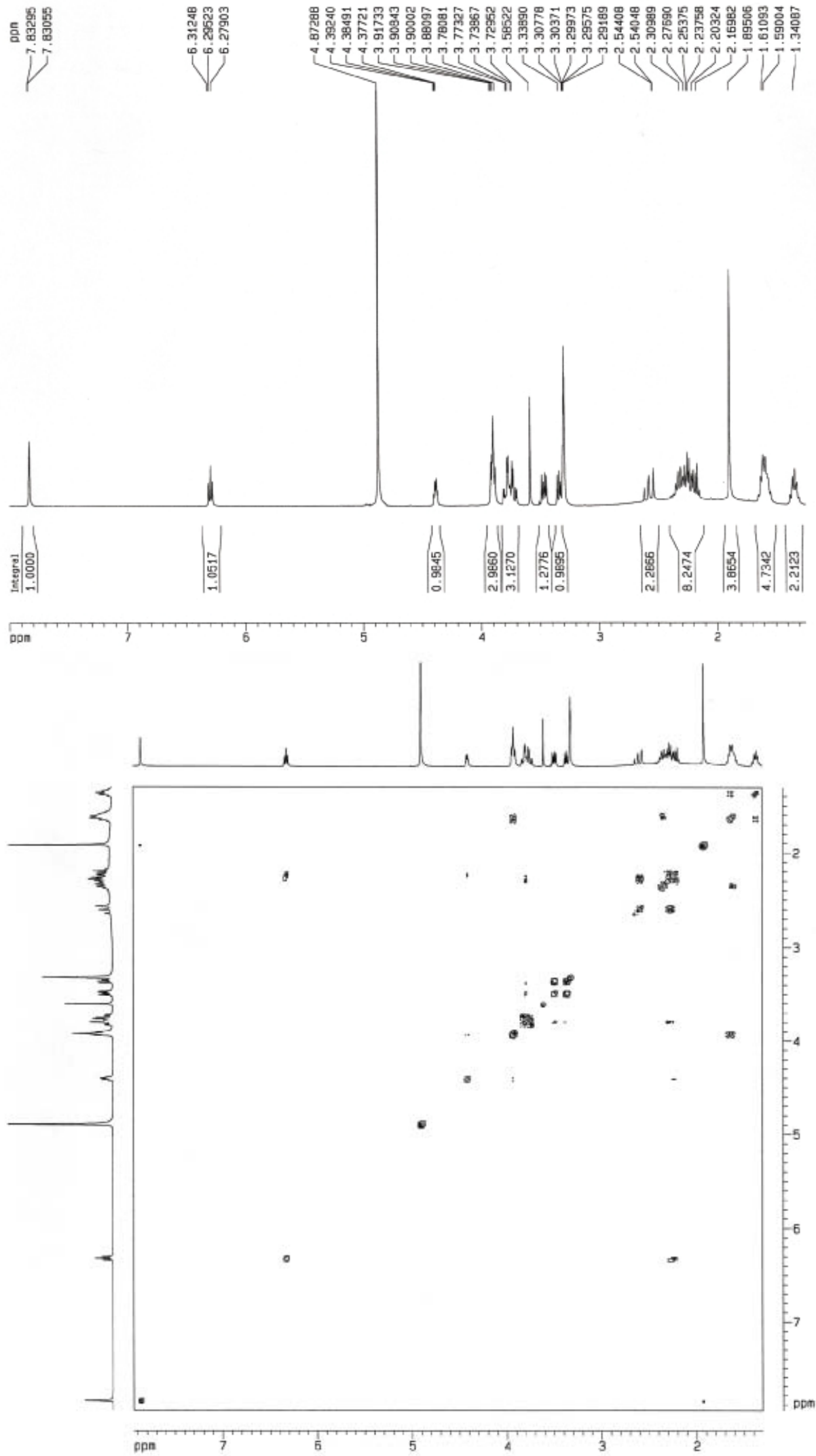

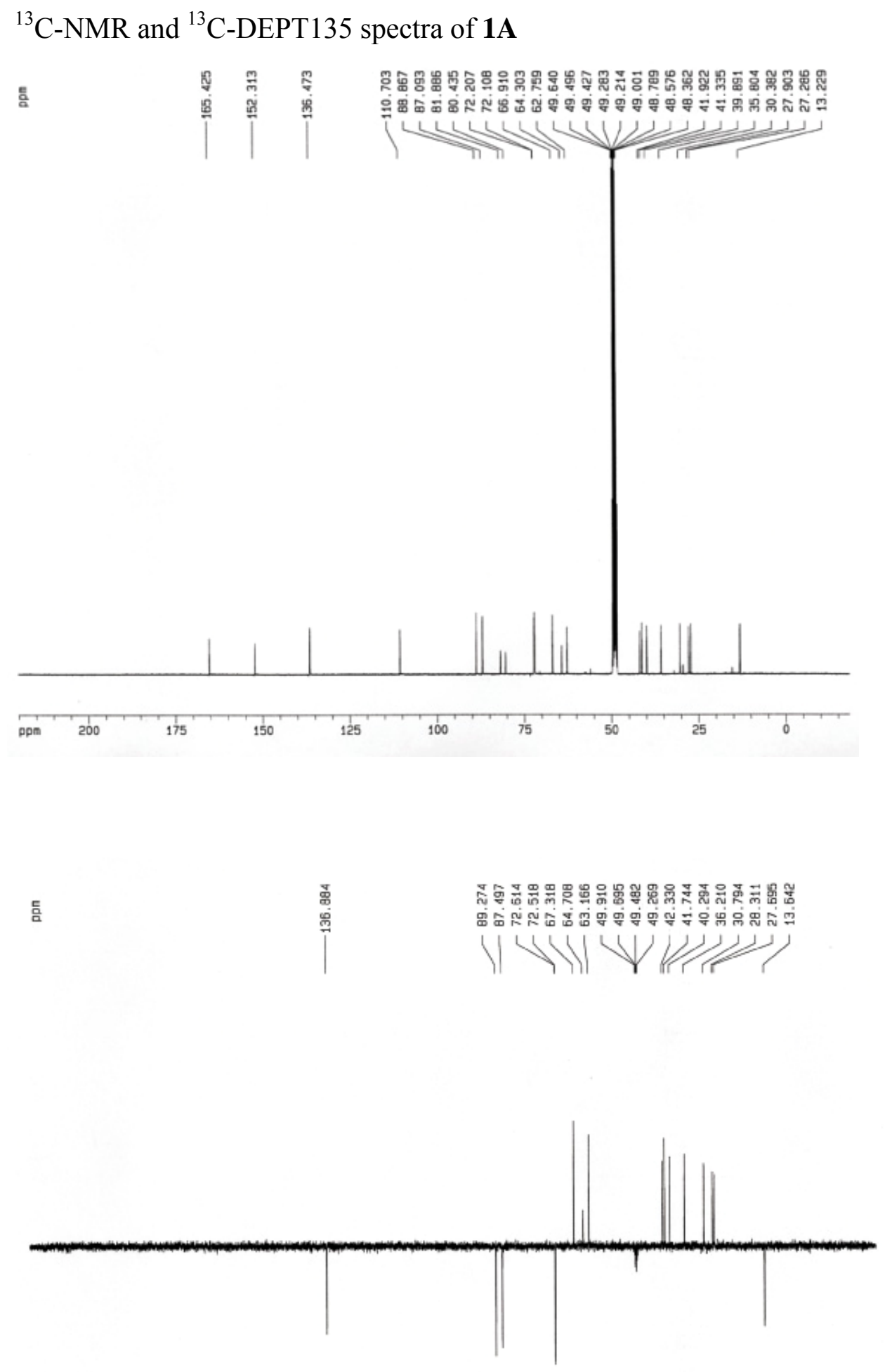

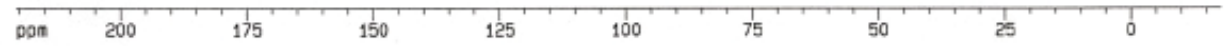


2D-HMQC spectrum of 1A

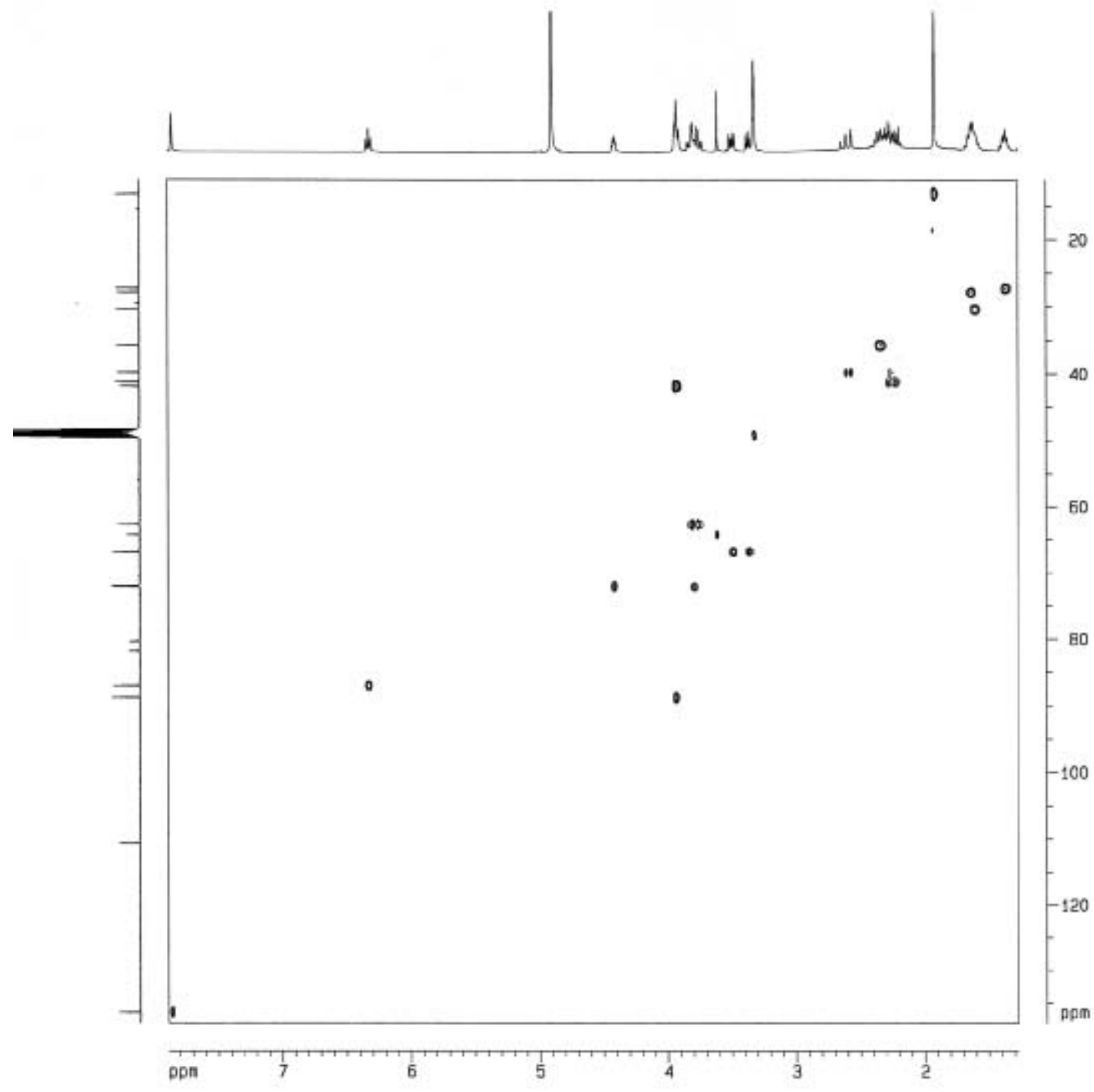


4. Scheme 1. Synthesis of compound 11, Experimental section

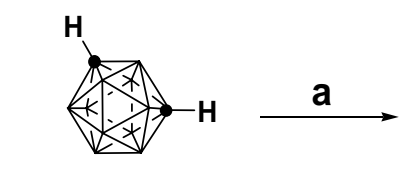

$m$-carborane
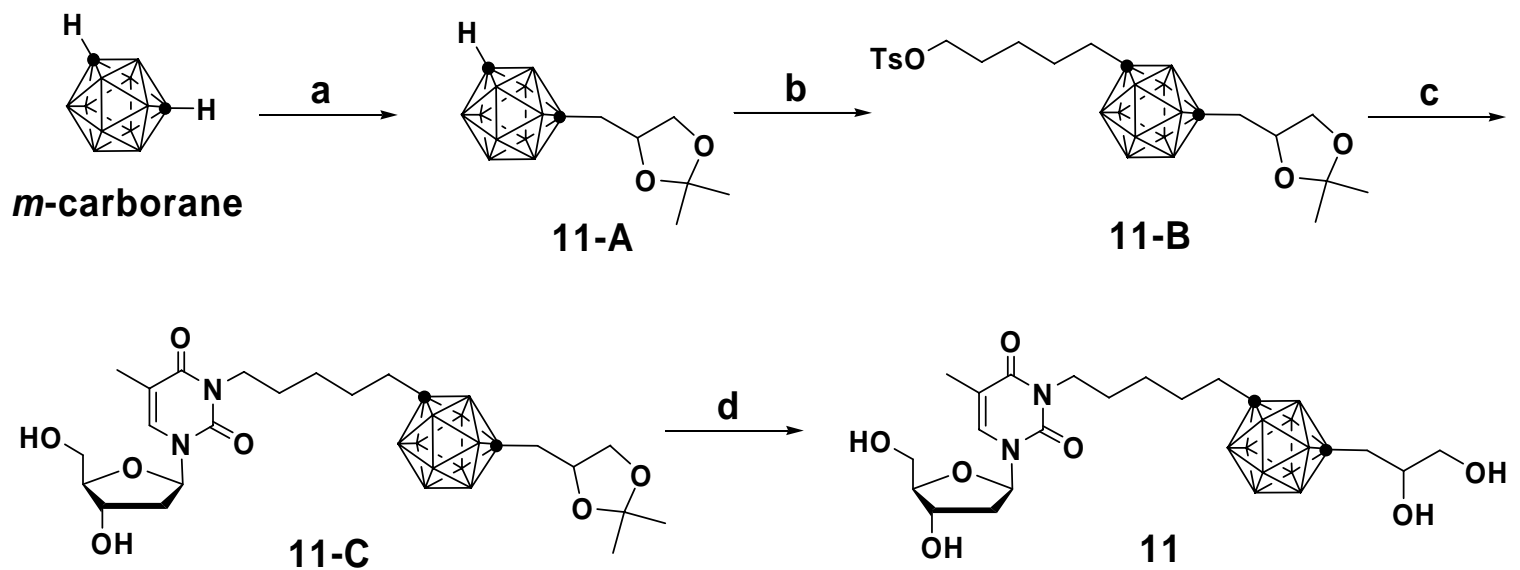

\section{4-(m-Carboran-1-yl)methyl-2,2-dimethyl-1,3-dioxolane (11-A)}

To a solution of $m$-carborane $(426 \mathrm{mg}, 3.0 \mathrm{mmol})$ in benzene $(10 \mathrm{~mL})$ was added a solution of n-butylithium $\left(1.32 \mathrm{~mL}, 3.3 \mathrm{mmol}, 2.5 \mathrm{M}\right.$ solution in hexanes) at $0{ }^{\circ} \mathrm{C}$. The solution was first stirred for $30 \mathrm{~min}$ at $0{ }^{\circ} \mathrm{C}$ and subsequently for $30 \mathrm{~min}$ at room temperature. The reaction mixture was again cooled to $0{ }^{\circ} \mathrm{C}$ and 2,2-dimethyl-1,3dioxolane-4-ylmethyl p-tosylate $(858 \mathrm{mg}, 3.0 \mathrm{mmol})$ in benzene $(10 \mathrm{~mL})$ was added dropwise. The reaction mixture was stirred at room temperature for $14 \mathrm{hrs}$. Subsequently, distilled water $(10 \mathrm{~mL})$ was added and excess benzene was removed under reduced pressure. The residue was extracted with ethylacetate $(50 \mathrm{~mL})$, the organic layer was washed with d-HCl solution $(20 \mathrm{~mL})$ and brine $(20 \mathrm{~mL})$ and dried over $\mathrm{MgSO}_{4}$. After filtration and evaporation, the residue was purified by silica gel columns chromatography using hexanes/ ethylacetate (15:1) as the eluent to give compound 11-A (456 mg, 59\%). $R_{f} 0.16 ;{ }^{1} \mathrm{H}-\mathrm{NMR}\left(\mathrm{CDCl}_{3}\right) \delta 1.30\left(\mathrm{~s}, 3 \mathrm{H}, \mathrm{CH}_{3}\right), 1.34\left(\mathrm{~s}, 3 \mathrm{H}, \mathrm{CH}_{3}\right), 2.07\left(\mathrm{dd}, 1 \mathrm{H}, \mathrm{CH}_{2^{-}}\right.$ $\left.\mathrm{C}_{\text {carborane, }} J=14.7,5.3 \mathrm{~Hz}\right), 2.29\left(\mathrm{dd}, 1 \mathrm{H}, \mathrm{CH}_{2}-\mathrm{C}_{\text {carborane }}, J=14.7,5.6 \mathrm{~Hz}\right), 2.92($ br s, $1 \mathrm{H}$, $\left.\underline{\mathrm{H}}-\mathrm{C}_{\text {carborane }}\right), 3.42-3.48\left(\mathrm{~m}, 1 \mathrm{H}, \mathrm{CH}_{2}\right), 4.01-4.07(\mathrm{~m}, 2 \mathrm{H}) ;{ }^{13} \mathrm{C}-\mathrm{NMR}\left(\mathrm{CDCl}_{3}\right) \delta 25.35$ 
$\left(\underline{\mathrm{CH}}_{3}\right), 26.86\left(\underline{\mathrm{CH}}_{3}\right), 41.18\left(\underline{\mathrm{CH}}_{2}-\mathrm{C}_{\text {carborane }}\right), 55.26\left(\mathrm{H}-\underline{\mathrm{C}}_{\text {carborane }}\right), 69.00\left(\underline{\mathrm{CH}}_{2}\right), 72.59\left(\mathrm{CH}_{2^{-}}\right.$

$\left.\underline{\mathrm{C}}_{\text {carborane }}\right), 74.66(\underline{\mathrm{C}} \mathrm{H}), 109.13\left[\underline{\mathrm{C}}\left(\mathrm{CH}_{3}\right)_{2}\right]$

\section{5-[2-(2,3-Isopropylidenedioxyprop-1-yl)-m-carboran-1-yl]pentyl tosylate (11-B)}

To a solution of compound 11-A (97 mg, $0.39 \mathrm{mmol})$ in benzene $(5 \mathrm{~mL})$ was added $\mathrm{n}$ butyllithium ( $0.19 \mathrm{~mL}, 0.47 \mathrm{mmol}, 2.5 \mathrm{M}$ solution in hexanes) at $5{ }^{\circ} \mathrm{C}$ over a period of 20 minutes. The solution was stirred at the same temperature for $30 \mathrm{~min}$ and then stirred at room temperature for $30 \mathrm{~min}$. The solution was slowly added to a solution of 1,5pentanediol di-p-tosylate $(254 \mathrm{mg}, 0.62 \mathrm{mmol})$ in benzene $(10 \mathrm{~mL})$ at $5{ }^{\circ} \mathrm{C}$ (ice bath). The reaction mixture was stirred for $1 \mathrm{hr}$ at the same temperature. Distilled water $(5 \mathrm{~mL})$ was added and the reaction mixture was extracted with ethylacetate $(10 \mathrm{~mL} \times 3)$. The combined organic layers were washed with brine $(10 \mathrm{~mL})$ and dried over magnesium sulfate. After filtration and evaporation, the residue was purified by silica gel column chromatography using hexanes/ethylacetate (4:1) as the eluent to give compound 11-B (125 mg, $64 \%) . R_{f} 0.21 ;{ }^{1} \mathrm{H}-\mathrm{NMR}\left(\mathrm{CDCl}_{3}\right) \delta 1.20-1.28\left(\mathrm{~m}, 4 \mathrm{H}, \mathrm{CH}_{2}\right), 1.30\left(\mathrm{~s}, 3 \mathrm{H}, \mathrm{CH}_{3}\right)$, $1.34\left(\mathrm{~s}, 3 \mathrm{H}, \mathrm{CH}_{3}\right), 1.55-1.60\left(\mathrm{~m}, 2 \mathrm{H}, \mathrm{CH}_{2}\right), 1.81-1.85$ (m, $\left.2 \mathrm{H}, \mathrm{C}_{2}-\mathrm{C}_{\text {carborane }}\right), 2.04$ (dd, $\left.1 \mathrm{H}, \underline{\mathrm{C}}_{2}-\mathrm{C}_{\text {carborane }}, J=14.7,5.4 \mathrm{~Hz}\right), 2.27\left(\mathrm{dd}, 1 \mathrm{H}, \underline{\mathrm{C}}_{2}-\mathrm{C}_{\text {carborane }}, J=14.7,5.6 \mathrm{~Hz}\right), 2.44$ (s, $\left.3 \mathrm{H}, \mathrm{CH}_{3}\right), 3.42-3.48\left(\mathrm{~m}, 1 \mathrm{H}, \mathrm{CH}_{2}\right), 3.97\left(\mathrm{t}, 2 \mathrm{H}, \mathrm{OCH}_{2}, J=6.3 \mathrm{~Hz}\right), 4.00-4.05(\mathrm{~m}, 2 \mathrm{H})$, $7.32(\mathrm{~d}, 2 \mathrm{H}, \mathrm{ArH}, J=8.2 \mathrm{~Hz}), 7.75(\mathrm{~d}, 2 \mathrm{H}, \mathrm{ArH}, J=8.2 \mathrm{~Hz}),{ }^{13} \mathrm{C}-\mathrm{NMR}\left(\mathrm{CDCl}_{3}\right) \delta 21.64$ $\left.\left(\underline{\mathrm{CH}}_{3}\right), 24.67\left(\underline{\mathrm{CH}}_{2}\right), 25.36(\underline{\mathrm{CH}})_{3}\right), 26.88\left(\underline{\mathrm{CH}}_{3}\right), 28.38\left(\underline{\mathrm{CH}}_{2}\right), 29.16\left(\underline{\mathrm{CH}}_{2}\right), 36.62\left(\underline{\mathrm{CH}}_{2}-\right.$

$\left.\mathrm{C}_{\text {carborane }}\right), 41.18\left(\underline{\mathrm{CH}}_{2}-\mathrm{C}_{\text {carborane }}\right), 69.02\left(\underline{\mathrm{C}} \mathrm{H}_{2}\right), 70.2\left(\underline{\mathrm{CH}}_{2} \mathrm{OTs}\right), 72.06$ (C- $\left.\underline{\mathrm{C}}_{\text {carborane }}\right), 74.65$ $(\underline{\mathrm{C}} \mathrm{H}), 76.06$ (C- $\left.\underline{\mathrm{C}}_{\text {carborane }}\right), 109.08\left(\underline{\mathrm{C}}\left(\mathrm{CH}_{3}\right)_{2}\right), 127.84$ (Ar-C), 129.84 (Ar-C), 133.01 (ArC), 144.78 (Ar-C); MS (HR-ESI) $\mathrm{C}_{20} \mathrm{H}_{38} \mathrm{~B}_{10} \mathrm{O}_{5} \mathrm{~S}_{1} \mathrm{Na}(\mathrm{M}+\mathrm{Na})^{+}$calcd 521.3352, found 521.3336 . 


\section{3-\{5-[2-(2,3-Isopropylidenedioxyprop-1-yl)-m-carboran-1-yl]pentan-1-yl\}thymidine}

\section{(11-C)}

To a solution of compound 11-B (197 mg, $0.40 \mathrm{mmol})$ in a dimethylformamide-acetone mixture $(20 \mathrm{~mL}, 1: 1)$ was added thymidine $(239 \mathrm{mg}, 0.99 \mathrm{mmol})$ and potassium carbonate $(150 \mathrm{mg}, 1.13 \mathrm{mmol})$. The reaction mixture was stirred at $50{ }^{\circ} \mathrm{C}$ for $48 \mathrm{hr}$. The reaction mixture was filtered and the filtrate was concentrated under reduced pressure. The residue was purified by silica gel columns chromatography using ethylacetate/methanol $(25: 1)$ as the eluent to give compound 11-C (130 mg, 57\%). $R_{f}$ 0.25; ${ }^{1} \mathrm{H}-\mathrm{NMR}$ (Acetone- $\left.\mathrm{d}^{6}\right) \delta$ 1.20-1.24 (m, 2H, $\left.\mathrm{CH}_{2}\right) 1.27\left(\mathrm{~s}, 3 \mathrm{H}, \mathrm{CH}_{3}\right), 1.30(\mathrm{~s}, 3 \mathrm{H}$, $\left.\mathrm{CH}_{3}\right), 1.37-1.45\left(\mathrm{~m}, 2 \mathrm{H}, \mathrm{CH}_{2}\right), 1.50-1.58\left(\left(\mathrm{~m}, 2 \mathrm{H}, \mathrm{CH}_{2}\right), 1.82\left(\mathrm{~s}, 3 \mathrm{H}, \mathrm{CH}_{3}\right), 1.95-2.00(\mathrm{~m}\right.$, 2H, H-2 ), 2.21-2.24 (m, 4H, $\left.\mathrm{CH}_{2}-\mathrm{C}_{\text {carborane }}\right), 3.44\left(\mathrm{dd}, 1 \mathrm{H}, \mathrm{CH}_{2}, J=7.5,5.8 \mathrm{~Hz}\right), 3.76-$ $3.85\left(\mathrm{~m}, 4 \mathrm{H}, \mathrm{CH}_{2} \mathrm{~N}\right.$ and H-5'), $3.92(\mathrm{~m}, 1 \mathrm{H}, \mathrm{H}-4)$ ), 4.02-4.10 (m, 2H, $\left.\mathrm{CH}_{2}\right), 4.23(\mathrm{t}, 1 \mathrm{H}$,

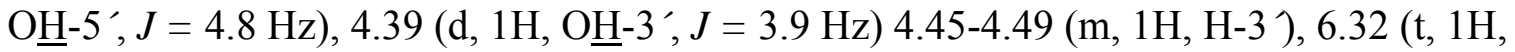
H-1', $J=6.7 \mathrm{~Hz}), 7.81(\mathrm{~s}, 1 \mathrm{H}, \mathrm{H}-6) ;{ }^{13} \mathrm{C}-\mathrm{NMR}$ (Acetone-d $\left.{ }^{6}\right) \delta 13.28\left(\underline{\mathrm{CH}}_{3}\right), 25.76\left(\underline{\mathrm{CH}}_{3}\right)$, $\left.\left.26.93\left(\underline{\mathrm{CH}}_{3}\right), 27.10(\underline{\mathrm{CH}})_{2}\right), 27.75\left(\underline{\mathrm{CH}}_{2}\right), 30.32(\underline{\mathrm{CH}})_{2}\right), 37.38\left(\underline{\mathrm{CH}}_{2}-\mathrm{C}_{\text {carborane }}\right), 41.13\left(\underline{\mathrm{CH}}_{2}-\right.$ $\left.\mathrm{C}_{\text {carborane }}\right), 41.89\left(\mathrm{C}-2^{\prime}\right), 42.92\left(\underline{\mathrm{CH}}_{2}-\mathrm{N}\right), 62.49\left(\mathrm{C}-5^{\prime}\right), 69.38\left(\underline{\mathrm{CH}}_{2}\right), 71.78\left(\mathrm{C}-3^{\prime}\right), 75.36$

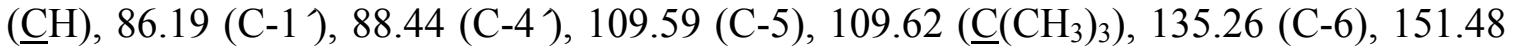
(C-2), 163.52 (C-4) ; MS (HR-ESI) $\mathrm{C}_{23} \mathrm{H}_{44} \mathrm{~B}_{10} \mathrm{~N}_{2} \mathrm{O}_{7} \mathrm{Na}(\mathrm{M}+\mathrm{Na})^{+}$calcd 591.4061, found 591.4046.

\section{3-\{5-[7-(2,3-Dihydroxyprop-1-yl)-m-carboran-1-yl]pentan-1-yl\}thymidine (11)}

To a solution of compound 11-C $(80 \mathrm{mg}, 0.14 \mathrm{mmol})$ in methanol $(8 \mathrm{~mL})$ was added a mixture of $3 \mathrm{~N}-\mathrm{HCl}$ and ethanol $(2 \mathrm{~mL}, 1: 1)$. The reaction mixture was stirred at room temperature for $20 \mathrm{hrs}$. Potassium carbonate $(35 \mathrm{mg}$ ) was added to the reaction mixture 
and was stirred for 30 mins at room temperature. The reaction mixture was filtered using Buchner funnel to remove the solid and the filtrate was concentrated under high vacuum pressure. The residue was purified by silica gel columns chromatography using ethylacetate/methanol (15:1) as the solvent system to give compound $11(65 \mathrm{mg}, 88 \%) . R_{f}$ 0.25; ${ }^{1} \mathrm{H}-\mathrm{NMR}\left(\mathrm{MeOH}-\mathrm{d}^{4}\right) \delta$ 1.21-1.28 (m, 2H, $\left.\mathrm{CH}_{2}\right), 1.38-1.44\left(\mathrm{~m}, 2 \mathrm{H}, \mathrm{CH}_{2}\right), 1.53-1.60$ (m, 2H, $\left.\mathrm{CH}_{2}\right), 1.89$ (d, 3H, $\left.\mathrm{CH}_{3}, J=1.1 \mathrm{~Hz}\right), 1.92-1.98$ (m, 2H, $\left.\underline{\mathrm{H}}_{2}-\mathrm{C}_{\text {carborane }}\right), 2.16-2.30$ (m, 4H, H-2' and $\underline{\mathrm{CH}}_{2}-\mathrm{C}_{\text {carborane }}$ ), $3.26\left(\mathrm{dd}, 1 \mathrm{H}, \underline{\mathrm{C}}_{2} \mathrm{OH}, J=11.1,6.1 \mathrm{~Hz}\right), 3.36(\mathrm{dd}, 1 \mathrm{H}$, $\left.\mathrm{C}_{2} \mathrm{OH}, J=11.1,5.5 \mathrm{~Hz}\right), 3.55-3.60\left(\mathrm{~m}, 1 \mathrm{H}, \mathrm{C} \underline{\mathrm{H}}(\mathrm{OH})-\mathrm{CH}_{2} \mathrm{OH}\right), 3.72\left(\mathrm{dd}, 1 \mathrm{H}, \mathrm{H}-5^{\prime}, J=\right.$ 12.1, 3.7 Hz), $3.79\left(\mathrm{dd}, 1 \mathrm{H}, \mathrm{H}^{-} 5^{\prime}, J=12.1,3.2 \mathrm{~Hz}\right), 3.86-3.92\left(\mathrm{~m}, 3 \mathrm{H}, \mathrm{H}^{\prime} 4^{\prime}\right.$ and $\left.\mathrm{CH}_{2} \mathrm{~N}\right)$, 4.37-4.40 (m, 1H, H-3), 6.29 (t, 1H, H-1', $J=6.8 \mathrm{~Hz}), 7.83$ (d, 1H, H-6, $J=1.1 \mathrm{~Hz})$; ${ }^{13} \mathrm{C}-\mathrm{NMR}\left(\mathrm{MeOH}-\mathrm{d}^{4}\right) \delta 13.22\left(\mathrm{CH}_{3}\right), 27.40\left(\mathrm{CH}_{2}\right), 28.05\left(\mathrm{CH}_{2}\right), 30.74\left(\mathrm{CH}_{2}\right), 37.98$ $\left(\mathrm{CH}_{2}\right), 41.34\left(\mathrm{CH}_{2}\right), 41.80\left(\mathrm{CH}_{2}\right), 42.01\left(\mathrm{CH}_{2}\right), 62.75\left(\mathrm{O}-\mathrm{CH}_{2}\right), 66.94\left(\mathrm{O}-\mathrm{CH}_{2}\right), 72.10(\mathrm{O}-$

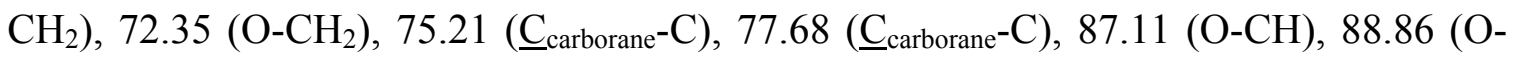
CH), 110.69 (C-5), 136.47 (C-6), 152.29 (C-2), 165.40 (C-4); MS (HR-ESI) $\mathrm{C}_{20} \mathrm{H}_{40} \mathrm{~B}_{10} \mathrm{~N}_{2} \mathrm{O}_{7} \mathrm{Na}(\mathrm{M}+\mathrm{Na})^{+}$calcd 551.3747, found 551.3740. 
5. Scheme 2. Synthesis of compound 12, Experimental section

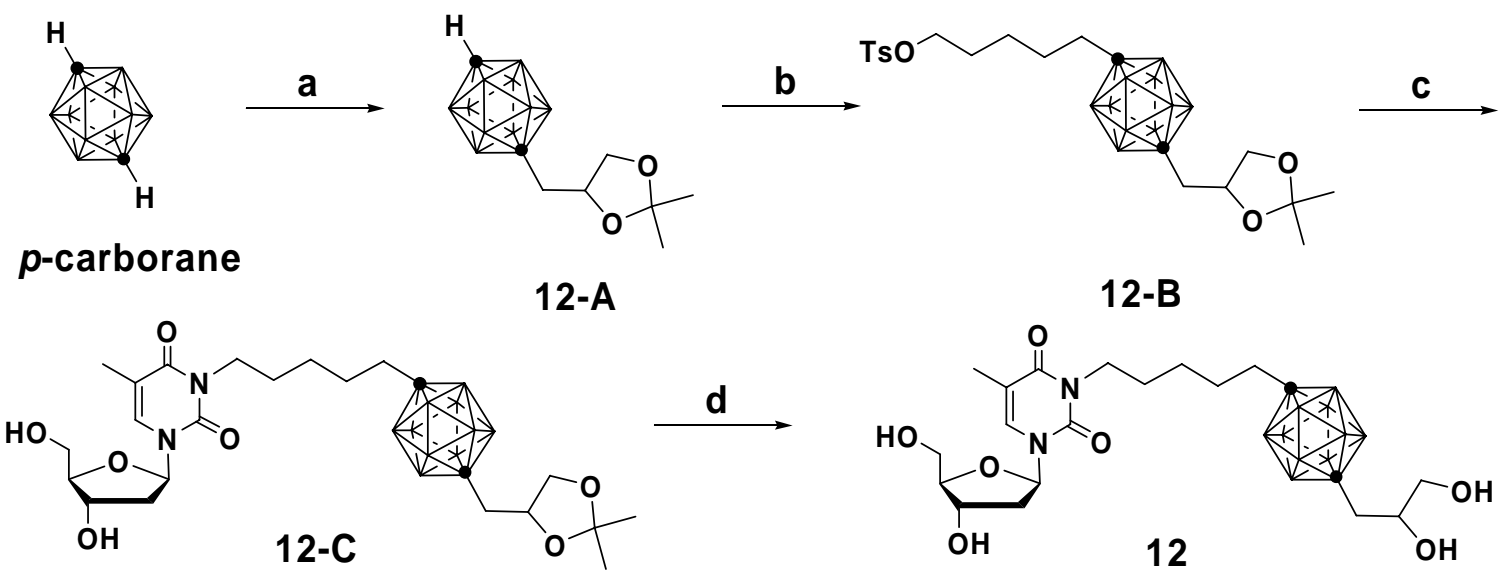

\section{4-(p-Carboran-1-yl)methyl-2,2-dimethyl-1,3-dioxolane (12-A)}

To a solution of p-carborane $(426 \mathrm{mg}, 3.0 \mathrm{mmol})$ in benzene $(10 \mathrm{~mL})$ was added a solution of n-butyllithium (1.32 mL, $3.3 \mathrm{mmol}, 2.5 \mathrm{M}$ solution in hexanes) at $0{ }^{\circ} \mathrm{C}$. The solution was first stirred for $30 \mathrm{~min}$ at $0{ }^{\circ} \mathrm{C}$ and subsequently for $30 \mathrm{~min}$ at room temperature. The reaction mixture was again cooled to $0{ }^{\circ} \mathrm{C}$ and 2,2-dimethyl-1,3dioxolane-4-ylmethyl p-tosylate $(858 \mathrm{mg}, 3.0 \mathrm{mmol})$ in benzene $(10 \mathrm{~mL})$ was added dropwise. The reaction mixture was stirred at room temperature for $14 \mathrm{hr}$. Subsequently, distilled water $(10 \mathrm{~mL})$ was added and excess benzene was removed under reduced pressure. The residue was extracted with ethylacetate $(50 \mathrm{~mL})$, the organic layer was washed with d-HCl solution $(20 \mathrm{~mL})$ and brine $(20 \mathrm{~mL})$ and dried over $\mathrm{MgSO}_{4}$. After filtration and evaporation, the residue was purified by silica gel columns chromatography using hexanes/ ethylacetate (15:1) as the eluent to give compound 11-A (386 mg, 50\%). $R_{f} 0.18 ;{ }^{1} \mathrm{H}-\mathrm{NMR}\left(\mathrm{CDCl}_{3}\right) \delta 1.26\left(\mathrm{~s}, 3 \mathrm{H}, \mathrm{CH}_{3}\right), 1.29\left(\mathrm{~s}, 3 \mathrm{H}, \mathrm{CH}_{3}\right), 1.76\left(\mathrm{dd}, 1 \mathrm{H}, \mathrm{CH}_{2^{-}}\right.$ $\left.\mathrm{C}_{\text {carborane, }} J=14.9,6.8 \mathrm{~Hz}\right), 1.98\left(\mathrm{dd}, 1 \mathrm{H}, \mathrm{CH}_{2}-\mathrm{C}_{\text {carborane }} J=14.9,5.4 \mathrm{~Hz}\right), 2.63($ br s, $1 \mathrm{H}$, $\left.\underline{\mathrm{H}}-\mathrm{C}_{\text {carborane }}\right), 3.34\left(\mathrm{dd}, 1 \mathrm{H}, \mathrm{CH}_{2}-\mathrm{C}_{\text {carborane }}, J=8.1,5.9 \mathrm{~Hz}\right), 3.81-3.86(\mathrm{~m}, 1 \mathrm{H}, \mathrm{CH}), 3.95$ 
$\left(\mathrm{dd}, 1 \mathrm{H}, \mathrm{CH}_{2}-\mathrm{C}_{\text {carborane, }} J=8.1,5.9 \mathrm{~Hz}\right) ;{ }^{13} \mathrm{C}-\mathrm{NMR}\left(\mathrm{CDCl}_{3}\right) \delta 25.71\left(\underline{\mathrm{CH}}_{3}\right), 27.21\left(\underline{\mathrm{CH}}_{3}\right)$, $43.42\left(\underline{\mathrm{CH}}_{2}-\mathrm{C}_{\text {carborane }}\right), 59.24$ (H- $\left.\underline{\mathrm{C}}_{\text {carborane }}\right), 69.42\left(\underline{\mathrm{CH}}_{2}\right), 74.81(\underline{\mathrm{CH}}), 81.33\left(\mathrm{CH}_{2}-\underline{\mathrm{C}}_{\text {carborane }}\right)$, $109.18\left[\underline{\mathrm{C}}\left(\mathrm{CH}_{3}\right)_{2}\right]$

\section{5-[2-(2,3-Isopropylidenedioxyprop-1-yl)-p-carboran-1-yl]pentyl tosylate (12-B)}

To a solution of compound 12-A (200 mg, $0.8 \mathrm{mmol})$ in benzene $(10 \mathrm{~mL})$ was added $\mathrm{n}$ butyllithium $\left(0.4 \mathrm{~mL}, 1.0 \mathrm{mmol}, 2.5 \mathrm{M}\right.$ solution in hexanes) at $5{ }^{\circ} \mathrm{C}$ over a period of 20 minutes. The solution was stirred at the same temperature for $30 \mathrm{~min}$ and then stirred at room temperature for $30 \mathrm{~min}$. The solution was slowly added to a solution of 1,5pentanediol di-p-tosylate $(510 \mathrm{mg}, 1.25 \mathrm{mmol})$ in benzene $(15 \mathrm{~mL})$ at $5{ }^{\circ} \mathrm{C}$ (ice bath). The reaction mixture was stirred for $1 \mathrm{hr}$ at the same temperature. Distilled water $(5 \mathrm{~mL})$ was added and the reaction mixture was extracted with ethylacetate $(15 \mathrm{~mL} \times 3)$. The combined organic layers were washed with brine $(15 \mathrm{~mL})$ and dried over magnesium sulfate. After filtration and evaporation, the residue was purified by silica gel column chromatography using hexanes/ethylacetate $(4: 1)$ as the eluent to give compound 12-B (260 mg, $65 \%$ ). $R_{f}$ 0.21; ${ }^{1} \mathrm{H}-\mathrm{NMR}\left(\mathrm{CDCl}_{3}\right) \delta$ 0.99-1.13 (m, 4H, $\left.\mathrm{CH}_{2}\right), 1.26\left(\mathrm{~s}, 3 \mathrm{H}, \mathrm{CH}_{3}\right)$, $1.29\left(\mathrm{~s}, 3 \mathrm{H}, \mathrm{CH}_{3}\right), 1.48-1.55\left(\mathrm{~m}, 4 \mathrm{H}, \mathrm{CH}_{2}\right.$ and $\left.\mathrm{C}_{2}-\mathrm{C}_{\text {carborane }}\right), 1.75$ (dd, $1 \mathrm{H}, \mathrm{C}_{2}-\mathrm{C}_{\text {carborane, }}$ $J=14.9,7.1 \mathrm{~Hz}), 1.98\left(\mathrm{dd}, 1 \mathrm{H}, \mathrm{CH}_{2}-\mathrm{C}_{\text {carborane }}, J=14.9,5.4 \mathrm{~Hz}\right), 2.43\left(\mathrm{~s}, 3 \mathrm{H}, \mathrm{CH}_{3}\right), 3.34$ (dd, $\left.1 \mathrm{H}, \mathrm{CH}_{2}-\mathrm{C}_{\text {carborane, }} J=8.2,6.1 \mathrm{~Hz}\right), 3.78-3.86(\mathrm{~m}, 1 \mathrm{H}, \mathrm{CH}), 3.92\left(\mathrm{t}, 2 \mathrm{H}, \mathrm{OCH}_{2}, J=\right.$ 6.4 Hz), 3.99-4.03 (m, 1H, $\left.\mathrm{CH}_{2}\right), 7.32(\mathrm{~d}, 2 \mathrm{H}, \mathrm{ArH}, J=8.2 \mathrm{~Hz}), 7.75(\mathrm{~d}, 2 \mathrm{H}, \mathrm{ArH}, J=$ $8.2 \mathrm{~Hz}) ;{ }^{13} \mathrm{C}-\mathrm{NMR}\left(\mathrm{CDCl}_{3}\right) \delta 21.63\left(\underline{\mathrm{CH}}_{3}\right), 24.82\left(\underline{\mathrm{CH}}_{2}\right), 25.30\left(\underline{\mathrm{CH}}_{3}\right), 26.81\left(\underline{\mathrm{CH}}_{3}\right), 28.66$ $\left(\underline{\mathrm{C}} \mathrm{H}_{2}\right), 30.90\left(\underline{\mathrm{CH}}_{2}\right), 37.45\left(\underline{\mathrm{CH}}_{2}-\mathrm{C}_{\text {carborane }}\right), 41.32\left(\underline{\mathrm{CH}_{2}-} \mathrm{C}_{\text {carborane }}\right), 69.04\left(\underline{\mathrm{CH}_{2}}\right), 70.03$

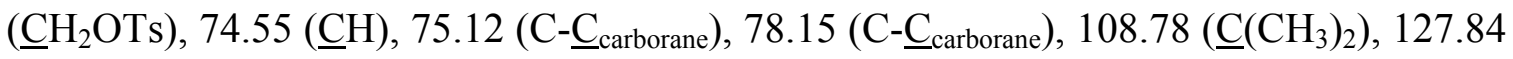


(Ar-C), 129.83 (Ar-C), 133.13 (Ar-C), 144.73 (Ar-C); MS (HR-ESI) $\mathrm{C}_{20} \mathrm{H}_{38} \mathrm{~B}_{10} \mathrm{O}_{5} \mathrm{~S}_{1} \mathrm{Na}$ $(\mathrm{M}+\mathrm{Na})^{+}$calcd 521.3352, found 521.3339.

\section{3-\{5-[2-(2,3-Isopropylidenedioxyprop-1-yl)-p-carboran-1-yl]pentan-1-yl\}thymidine} $(12-\mathrm{C})$

To a solution of compound 12-B (197 mg, $0.40 \mathrm{mmol})$ in a dimethylformamide-acetone mixture $(10 \mathrm{~mL}, 1: 1)$ was added thymidine $(245 \mathrm{mg}, 1.00 \mathrm{mmol})$ and potassium carbonate $(150 \mathrm{mg}, 1.13 \mathrm{mmol})$. The reaction mixture was stirred at $50^{\circ} \mathrm{C}$ for $48 \mathrm{hrs}$. The reaction mixture was filtered and the filtrate was concentrated under reduced pressure. The residue was purified by silica gel columns chromatography using ethylacetate/methanol (25:1) as eluent to give compound 11-C (140 mg, 62\%). $R_{f} 0.25$; ${ }^{1} \mathrm{H}-\mathrm{NMR}\left(\mathrm{MeOH}-\mathrm{d}^{4}\right) \delta$ 1.10-1.20 (m, 4H, $\left.\mathrm{CH}_{2}\right) 1.25\left(\mathrm{~s}, 3 \mathrm{H}, \mathrm{CH}_{3}\right), 1.27\left(\mathrm{~s}, 3 \mathrm{H}, \mathrm{CH}_{3}\right)$, 1.47-1.53 (m, $\left.2 \mathrm{H}, \mathrm{CH}_{2}\right), 1.60-1.64\left(\mathrm{~m}, 2 \mathrm{H}, \mathrm{CH}_{2}-\mathrm{C}_{\text {carborane }}\right), 1.78-1.93\left(\mathrm{~m}, 2 \mathrm{H}, \mathrm{CH}_{2}-\right.$ $\left.\mathrm{C}_{\text {carborane }}\right), 1.89\left(\mathrm{~d}, 3 \mathrm{H}, \mathrm{CH}_{3}, J=1.2 \mathrm{~Hz}\right), 2.14-2.29(\mathrm{~m}, 2 \mathrm{H}, \mathrm{H}-2), 3.30-3.32(\mathrm{~m}, 1 \mathrm{H}$, $\left.\mathrm{CH}_{2}\right), 3.70-3.95\left(\mathrm{~m}, 7 \mathrm{H}, \mathrm{CH}_{2} \mathrm{~N}, \mathrm{H}_{-} 5^{\prime}, \mathrm{CH}_{2}\right.$, and $\left.\mathrm{CH}\right), 4.36-4.40(\mathrm{~m}, 1 \mathrm{H}, \mathrm{H}-4), 6.28(\mathrm{t}, 1 \mathrm{H}$, $\left.\mathrm{H}-1^{\prime}, J=6.7 \mathrm{~Hz}\right), 7.82(\mathrm{~d}, 1 \mathrm{H}, \mathrm{H}-6, J=1.2 \mathrm{~Hz}) ;{ }^{13} \mathrm{C}-\mathrm{NMR}\left(\mathrm{MeOH}-\mathrm{d}^{4}\right) \delta 13.20\left(\underline{\mathrm{CH}_{3}}\right)$, $25.66\left(\underline{\mathrm{C}} \mathrm{H}_{3}\right), 27.16\left(\underline{\mathrm{CH}}_{3}\right), 27.32\left(\underline{\mathrm{C}} \mathrm{H}_{2}\right), 28.01\left(\underline{\mathrm{CH}}_{2}\right), 30.29\left(\underline{\mathrm{CH}}_{2}\right), 38.73\left(\underline{\mathrm{C}} \mathrm{H}_{2}-\mathrm{C}_{\text {carborane }}\right)$, $41.34\left(\underline{\mathrm{CH}}_{2}-\mathrm{C}_{\text {carborane }}\right), 41.99(\mathrm{C}-2), 42.96\left(\underline{\mathrm{CH}} \mathrm{H}_{2}-\mathrm{N}\right), 62.76(\mathrm{C}-5), 69.93\left(\underline{\mathrm{CH}}_{2}\right), 72.10(\mathrm{C}-$ 3), $75.89(\underline{\mathrm{C}} \mathrm{H}), 77.21$ (C- $\left.\underline{\mathrm{C}}_{\text {carborane }}\right), 81.43$ (C- $\left.\underline{\mathrm{C}}_{\text {carborane }}\right), 87.09$ (C-1'), 88.87 (C-4), 110.23 (C-5), $110.68\left(\underline{\mathrm{C}}\left(\mathrm{CH}_{3}\right)_{3}\right), 136.46$ (C-6), 152.28 (C-2), 165.39 (C-4); MS (HR-ESI) $\mathrm{C}_{23} \mathrm{H}_{44} \mathrm{~B}_{10} \mathrm{~N}_{2} \mathrm{O}_{7} \mathrm{Na}(\mathrm{M}+\mathrm{Na})^{+}$calcd 591.4061, found 591.4062 .

\section{3-\{5-[12-(2,3-Dihydroxyprop-1-yl)-p-carboran-1-yl]pentan-1-yl\}thymidine (12)}

To a solution of compound 12-C (100 mg, $0.18 \mathrm{mmol})$ in methanol $(10 \mathrm{~mL})$ was added a mixture of $3 \mathrm{~N}-\mathrm{HCl}$ and ethanol $(2 \mathrm{~mL}, 1: 1)$. The reaction mixture was stirred at room 
temperature for $20 \mathrm{hr}$. Potassium carbonate $(40 \mathrm{mg}$ ) was added to the reaction mixture and was stirred for $30 \mathrm{~min}$ at room temperature. The reaction mixture was filtered using Buchner funnel to remove the solid and the filtrate was concentrated under high vacuum pressure. The residue was purified by silica gel columns chromatography using ethylacetate/methanol (15:1) as the solvent system to give compound 12 (75 mg, 79\%). $R_{f}$ $0.25 ;{ }^{1} \mathrm{H}-\mathrm{NMR}\left(\mathrm{MeOH}-\mathrm{d}^{4}\right) \delta 1.10-1.20\left(\mathrm{~m}, 4 \mathrm{H}, \mathrm{CH}_{2}\right), 1.47-1.53\left(\mathrm{~m}, 2 \mathrm{H}, \mathrm{CH}_{2}\right), 1.60-1.66$ (m, 3H, $\left.\underline{\mathrm{H}}_{2}-\mathrm{C}_{\text {carborane }}\right), 1.89\left(\mathrm{~d}, 3 \mathrm{H}, \mathrm{CH}_{3}, J=1.1 \mathrm{~Hz}\right), 1.89-1.92\left(\mathrm{~m}, 1 \mathrm{H}, \mathrm{C}_{2}-\mathrm{C}_{\text {carborane }}\right)$, 2.14-2.29 (m, 2H, H-2 ), 3.16 (dd, 1H, $\left.\underline{\mathrm{C}}_{2} \mathrm{OH}, J=11.0,6.0 \mathrm{~Hz}\right), 3.24$ (dd, $1 \mathrm{H}, \underline{\mathrm{C}}_{2} \mathrm{OH}$, $J=11.0,5.6 \mathrm{~Hz}), 3.32-3.38(\mathrm{~m}, 1 \mathrm{H}, \mathrm{CH}), 3.71\left(\mathrm{dd}, 1 \mathrm{H}, \mathrm{H}-5^{\prime}, J=12.1,3.7 \mathrm{~Hz}\right), 3.79(\mathrm{dd}$, $\left.1 \mathrm{H}, \underline{\mathrm{C}}_{2} \mathrm{OH}, J=12.1,3.1 \mathrm{~Hz}\right), 3.83\left(\mathrm{t}, 2 \mathrm{H}, \mathrm{CH}_{2} \mathrm{~N}, J=7.6 \mathrm{~Hz}\right), 3.88-3.91(\mathrm{~m}, 1 \mathrm{H}, \mathrm{H}-3)$, 4.36-4.40 (m, 1H, H-4'), 6.28 (t, 1H, H-1', $J=6.8 \mathrm{~Hz}), 7.82(\mathrm{~d}, 1 \mathrm{H}, \mathrm{H}-6, J=1.1 \mathrm{~Hz})$; ${ }^{13} \mathrm{C}$ NMR $\left(\mathrm{CD}_{3} \mathrm{OD}\right) \delta 13.17\left(\mathrm{CH}_{3}\right), 27.33\left(\mathrm{CH}_{2}\right), 28.01\left(\mathrm{CH}_{2}\right), 30.27\left(\mathrm{CH}_{2}\right), 38.71\left(\mathrm{CH}_{2}\right)$, $41.34\left(\mathrm{CH}_{2}\right), 42.00\left(\mathrm{CH}_{2}\right), 42.52\left(\mathrm{CH}_{2}\right), 62.76\left(\mathrm{O}-\mathrm{CH}_{2}\right), 66.87\left(\mathrm{O}-\mathrm{CH}_{2}\right), 72.07\left(\mathrm{O}-\mathrm{CH}_{2}\right)$, $72.16\left(\mathrm{O}-\mathrm{CH}_{2}\right), 78.74\left(\underline{\mathrm{C}}_{\text {carborane-}} \mathrm{C}\right), 81.23\left(\underline{\mathrm{C}}_{\text {carborane }}{ }^{-\mathrm{C}}\right), 87.12(\mathrm{O}-\mathrm{CH}), 88.86(\mathrm{O}-\mathrm{CH})$, 110.69 (C-5), 136.43 (C-6), 152.28 (C-2), 165.38 (C-4); MS (HR-ESI) $\mathrm{C}_{20} \mathrm{H}_{40} \mathrm{~B}_{10} \mathrm{~N}_{2} \mathrm{O}_{7} \mathrm{Na}$ $(\mathrm{M}+\mathrm{Na})^{+}$calcd 551.3747, found 551.3746. 
6. Partial ${ }^{1} \mathrm{H}-\mathrm{NMR}$ spectra of 10A, 10B, 10C, and 1A with $(S)-(+)-2,2,2$-trifluoro-1- (9anthryl)-ethanol in $\mathrm{CDCl}_{3}$ at $300 \mathrm{~K}$
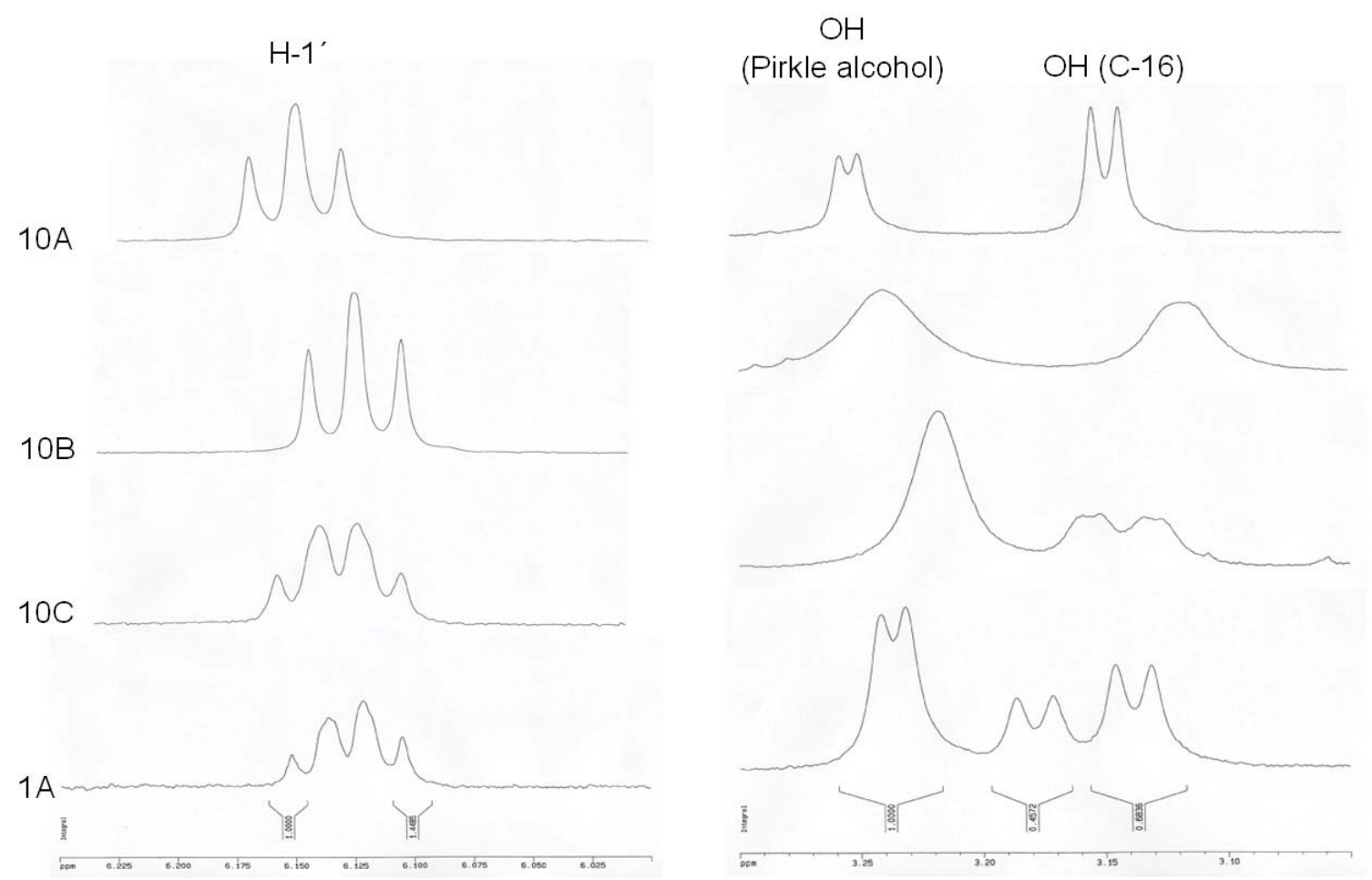
7. HPLC spectrum of a mixture of 10C, 11 and $\mathbf{1 2}$

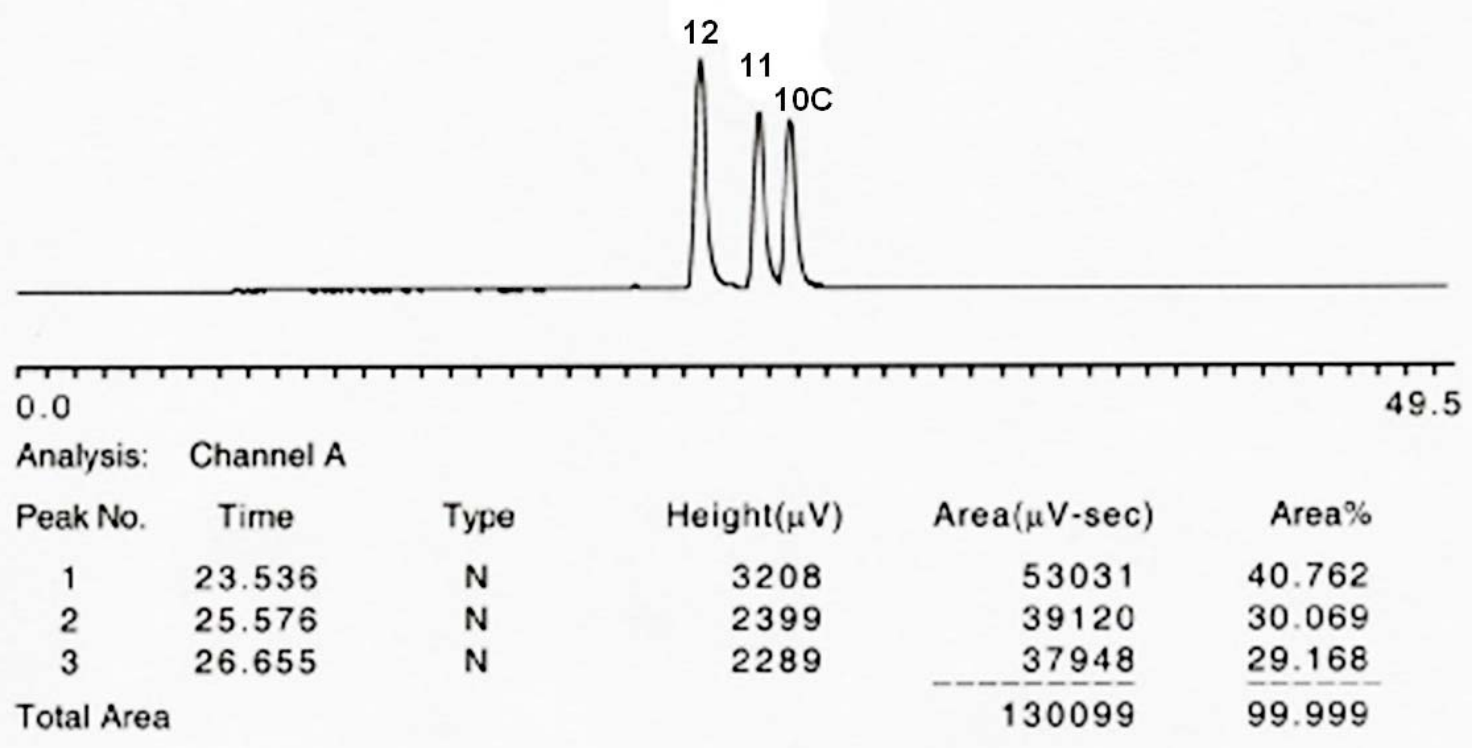


8. HPLC purity spectra of all target compounds using reversed-phase C-18. Mobile phases: Acetonitrile and water, methanol and water

\section{3-\{5-[2-(2,3-Dihydroxyprop-1-yl)-o-carboran-1-yl]pentan-1-yl\}thymidine (1A)}

Methanol/water gradient

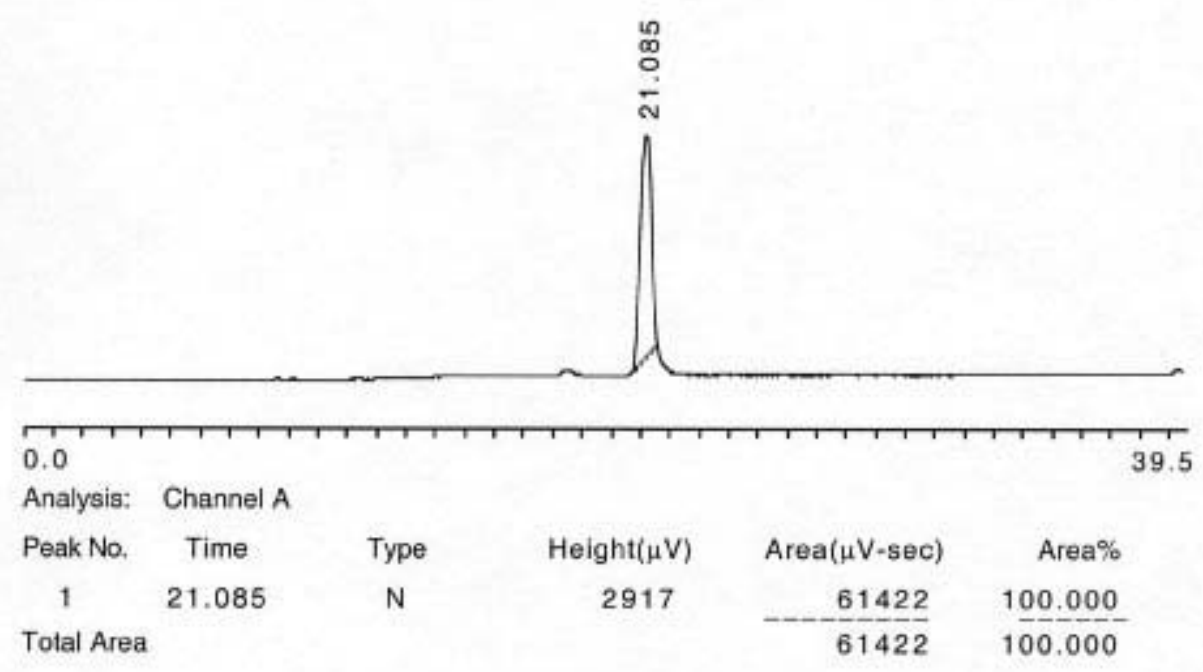

Acetonitrile/water gradient

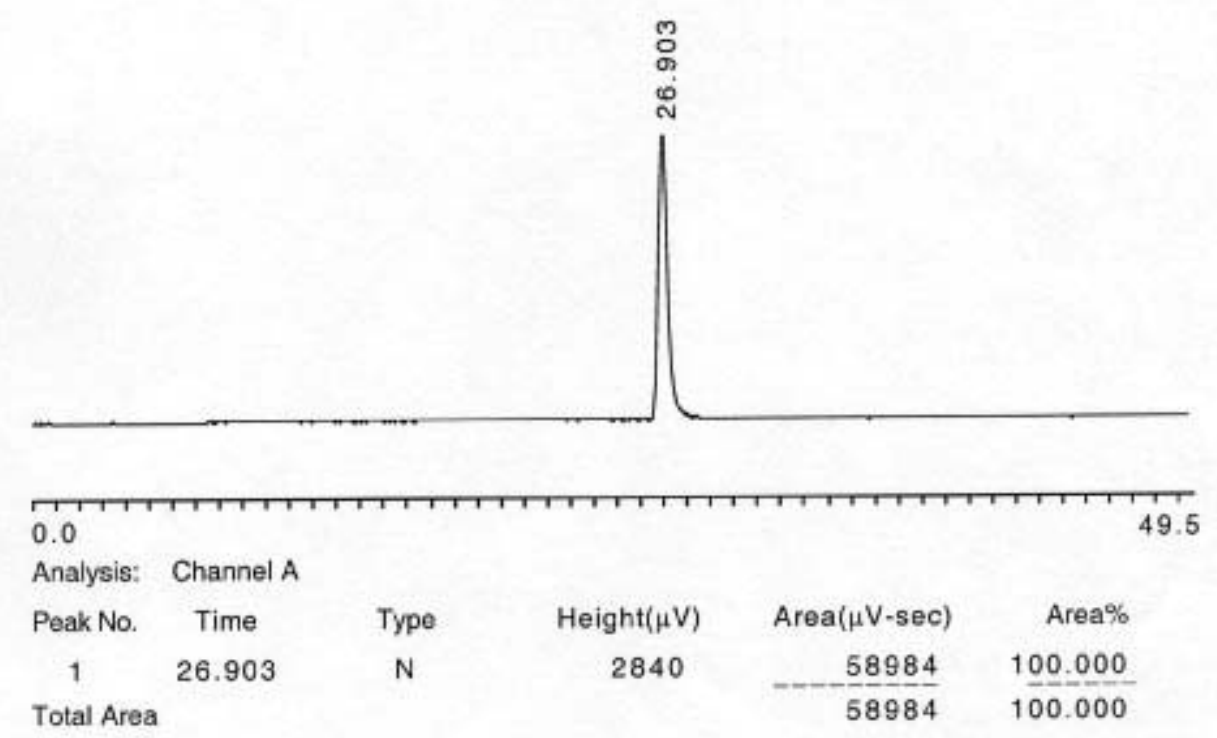




\section{3-\{5-[2-(2,3-Dihydroxyprop-1-yl)-o-carboran-1-yl]pentan-1-yl\}thymidine (1B)}

Methanol/water gradient

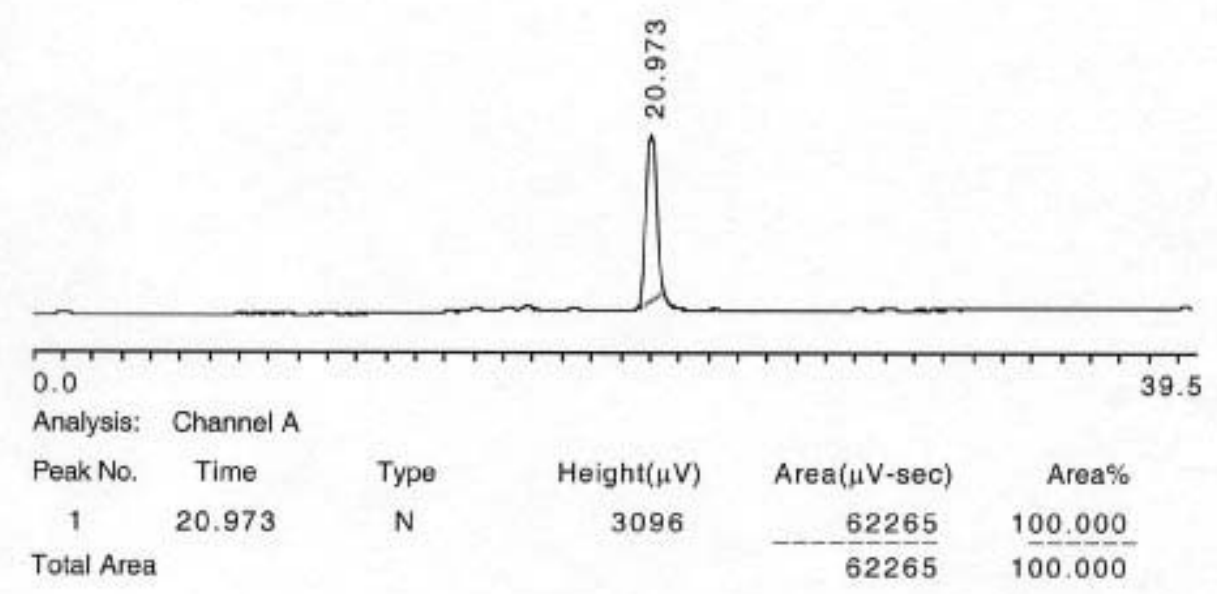

Acetonitrile/water gradient

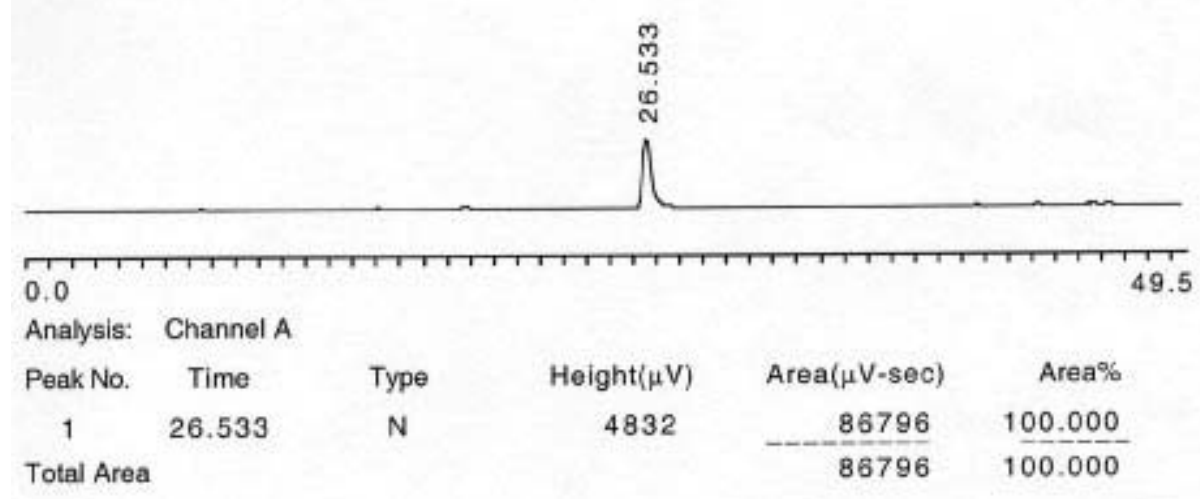


(R)-3-\{5-[2-(2,3-Dihydroxyprop-1-yl)-o-carboran-1-yl]pentan-1-yl\}thymidine (10A)

Methanol/water gradient

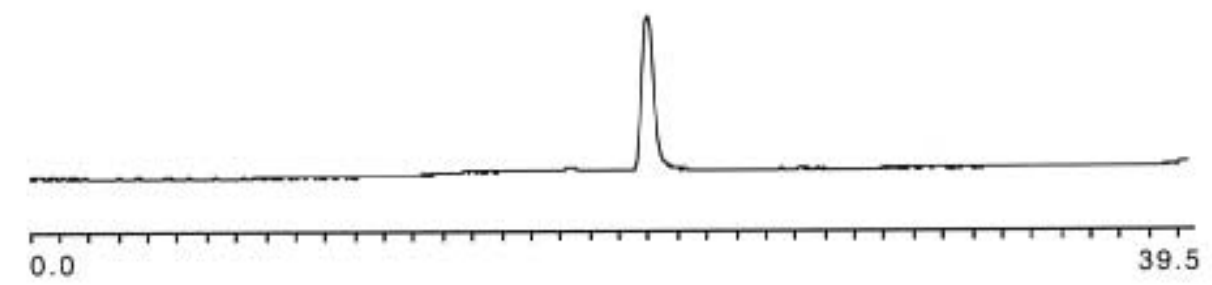

Acetonitrile/water gradient

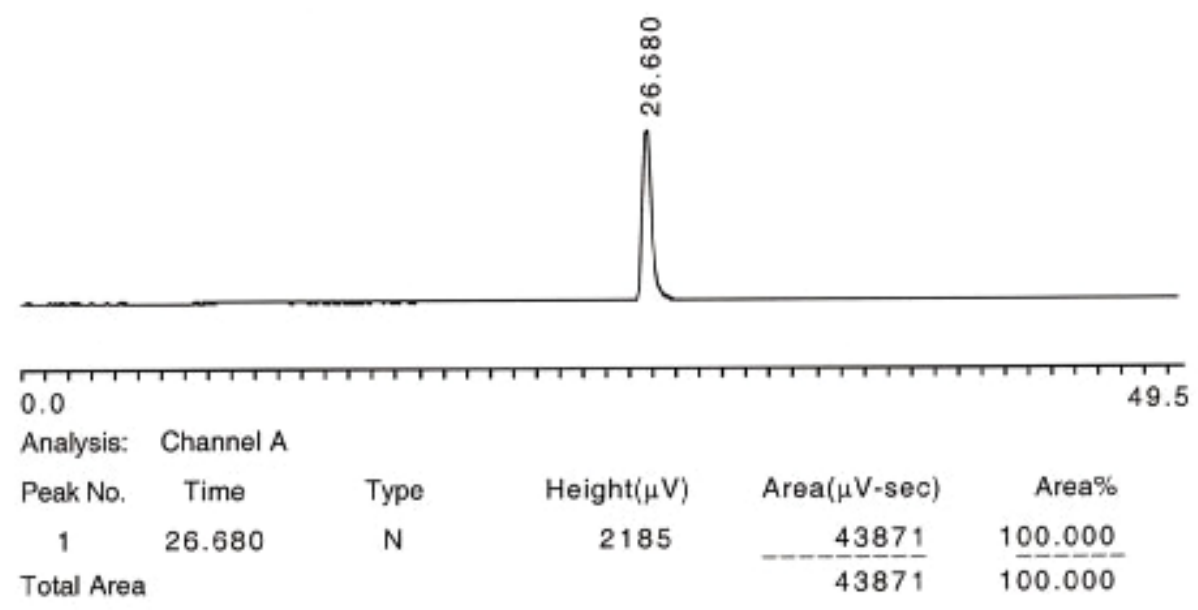


(S)-3-\{5-[2-(2,3-Dihydroxyprop-1-yl)-o-carboran-1-yl]pentan-1-yl\}thymidine (10B)

Methanol/water gradient

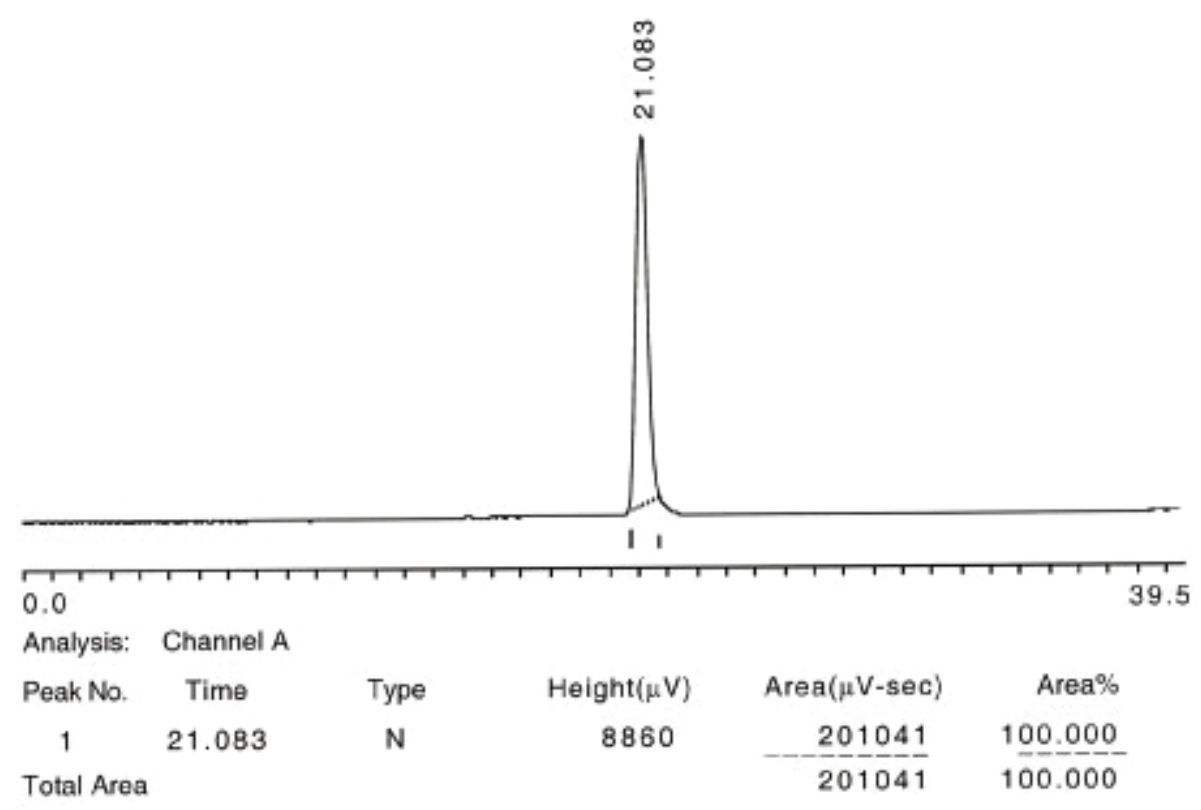

Acetonitrile/water gradient

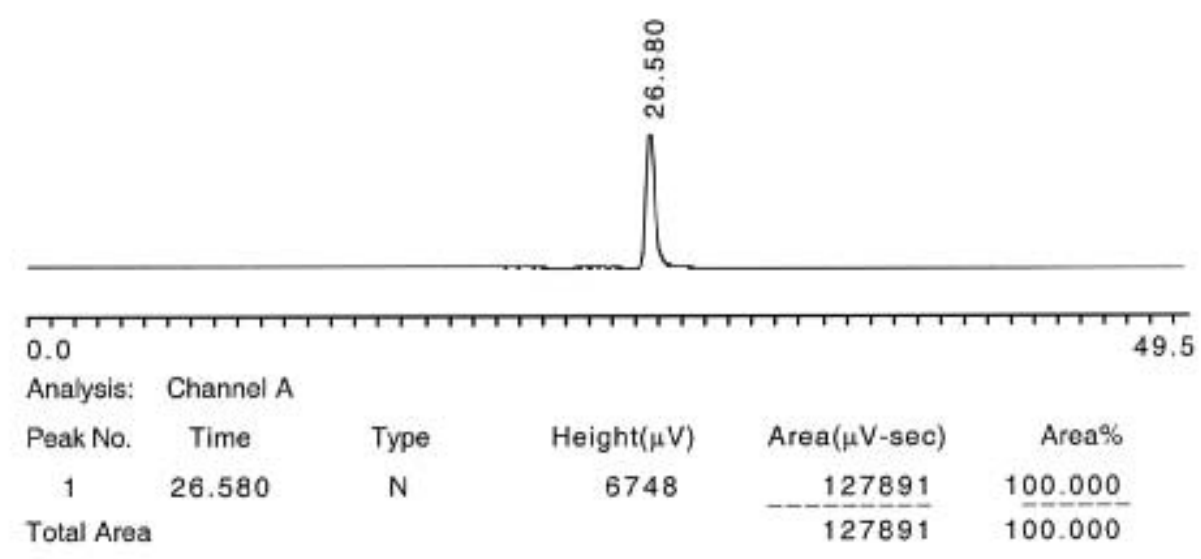




\section{3-\{5-[2-(2,3-Dihydroxyprop-1-yl)-o-carboran-1-yl]pentan-1-yl\}thymidine (10C)}

Methanol/water gradient

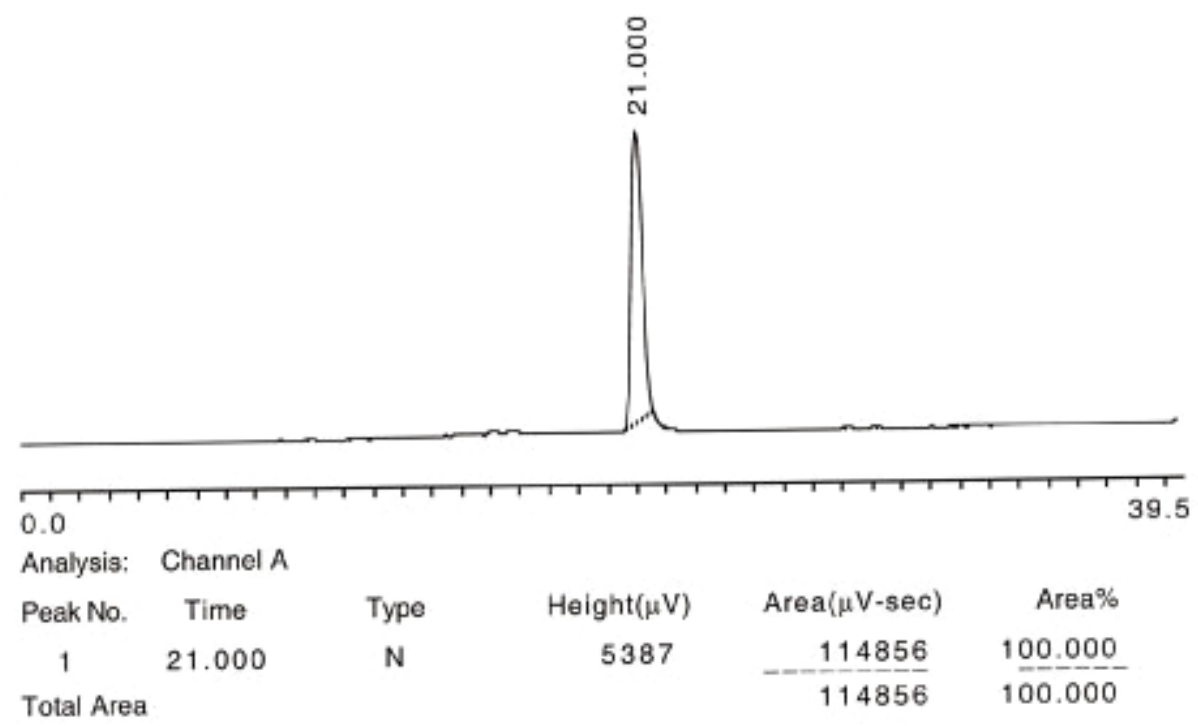

Acetonitrile/water gradient

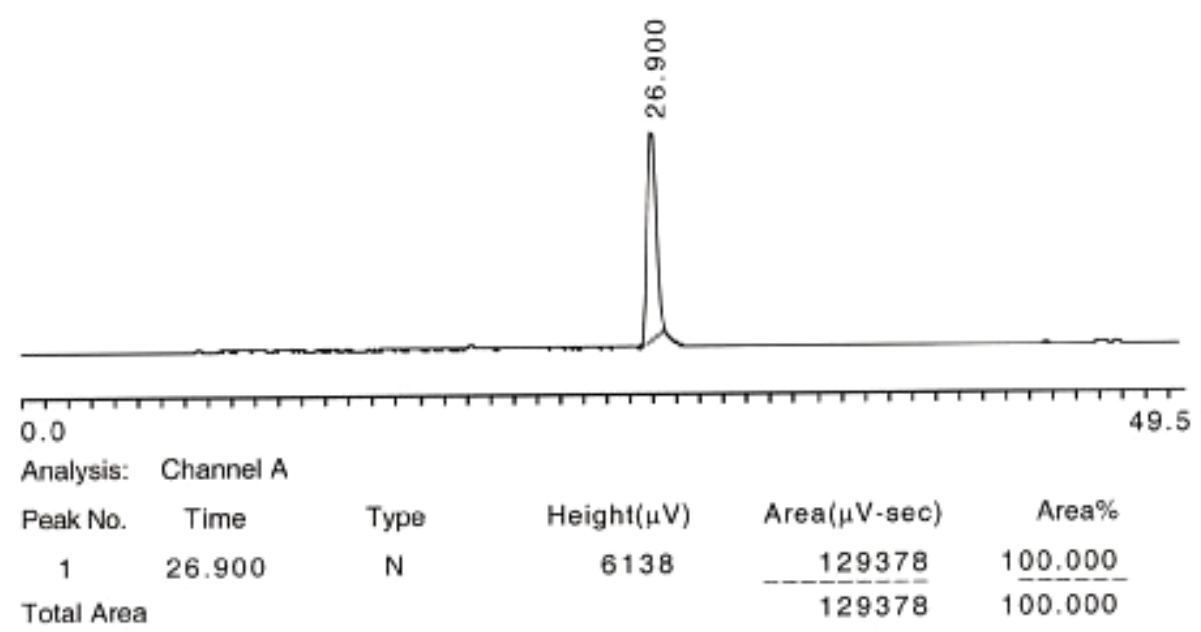


Methanol/water gradient

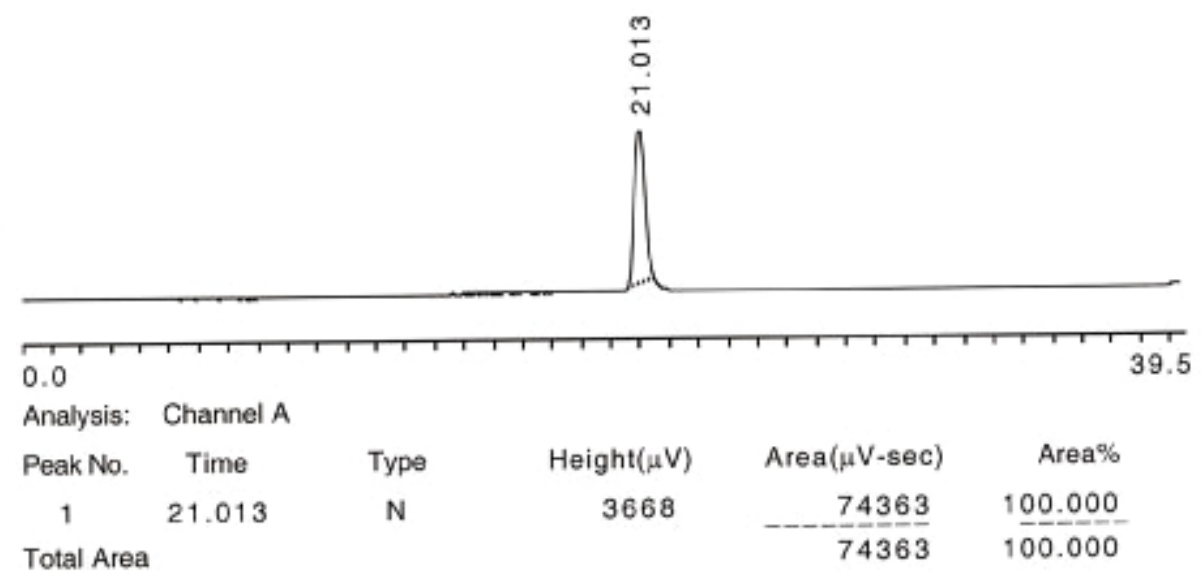

Acetonitrile/water gradient

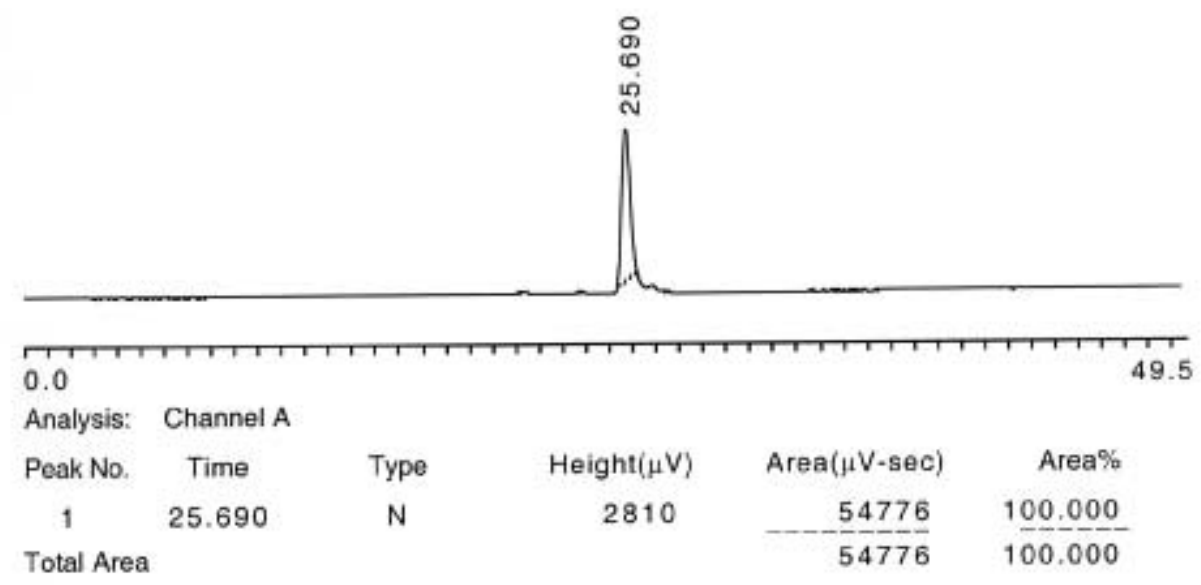


3-\{5-[2-(2,3-Dihydroxyprop-1-yl)-p-carboran-1-yl]pentan-1-yl\}thymidine (12)

Methanol/water gradient

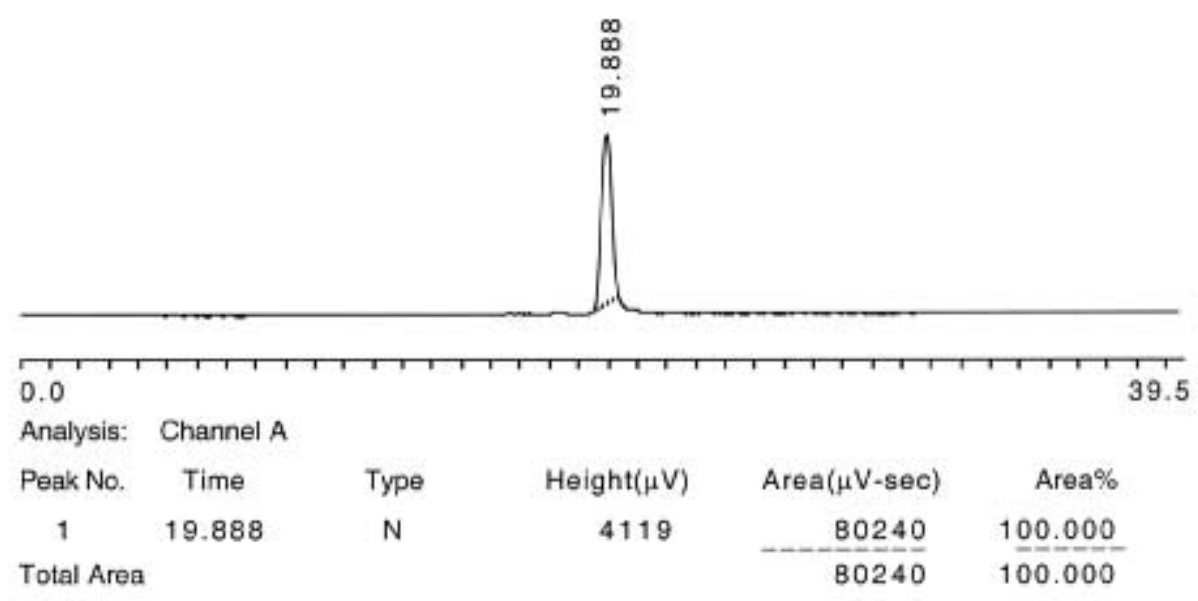

Acetonitrile/water gradient

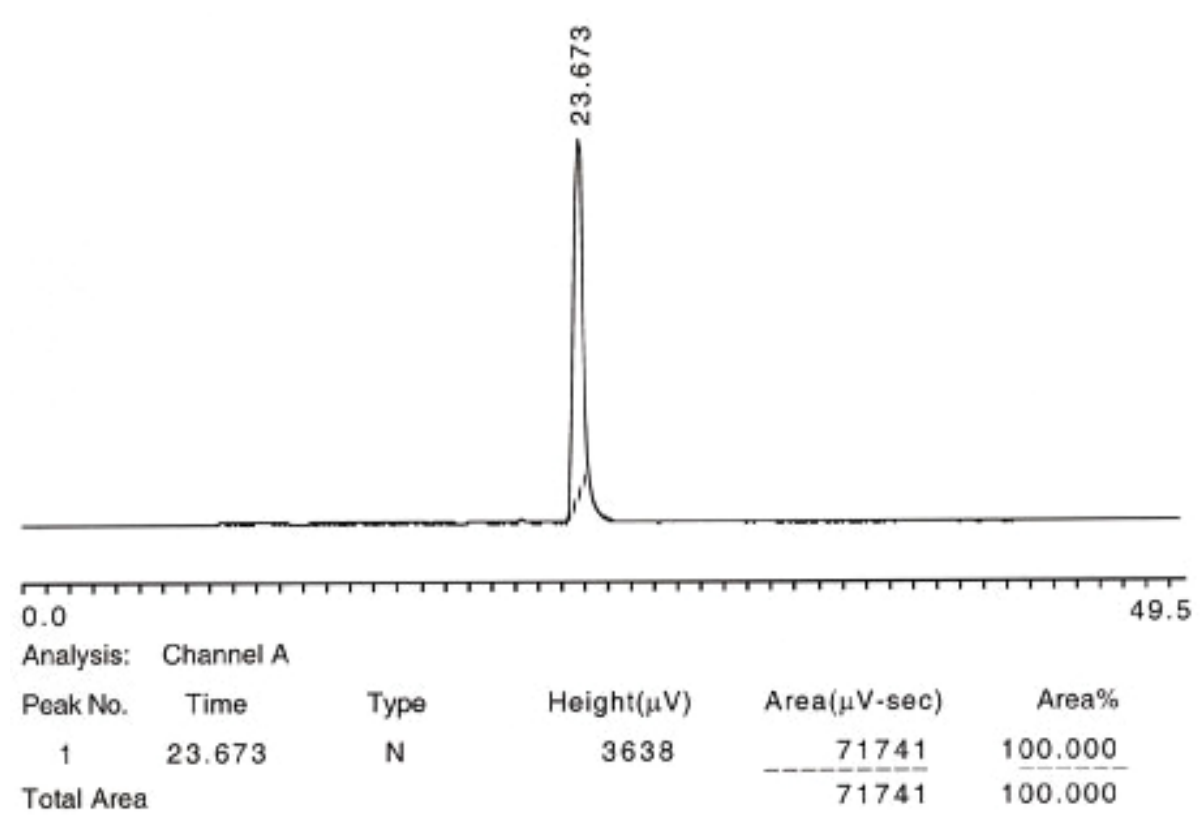


9. Calculated Energy, RMS gradient, and dipole moment of the minimized structures for 10A, (R)-epimers of $\mathbf{1 1}$ and $\mathbf{1 2}$ at B3LYP/6-31G* level using Gaussian 03 program.
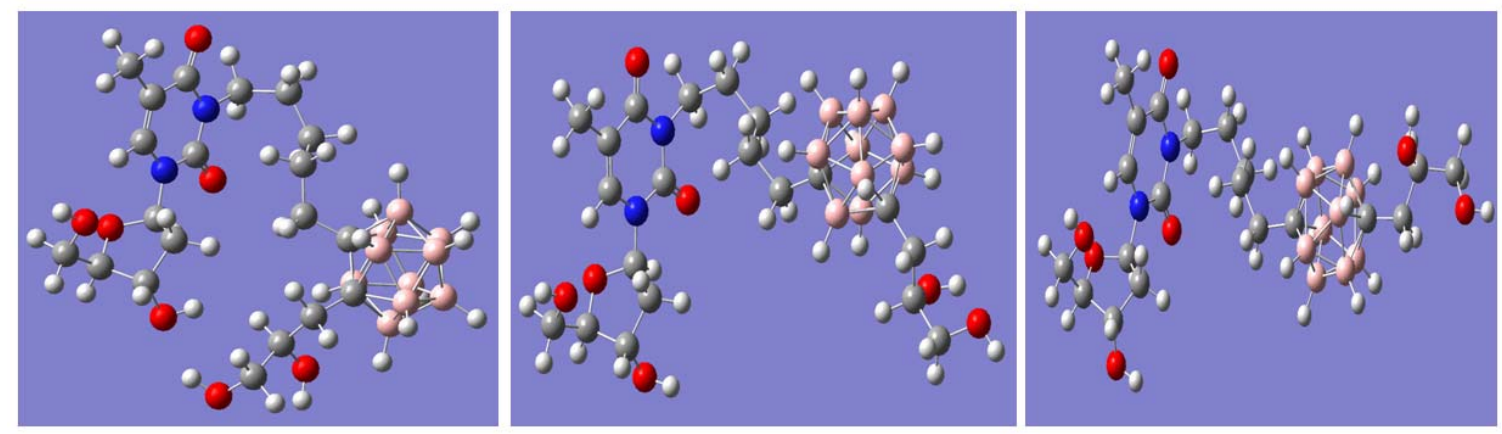

\begin{tabular}{cccc}
\hline & $\mathbf{1 0 A}$ & $\mathbf{1 1}^{a}$ & $\mathbf{1 2}^{a}$ \\
\hline E(RB+HF-LYP) & -1671.0231 a.u. & -1671.0347 a.u. & -1671.0331 a.u. \\
RMS gradient & $5.91 \times 10^{-6}$ a.u. & $1.60 \times 10^{-6}$ a.u. & $6.45 \times 10^{-6}$ a.u. \\
Dipole moment & 9.4599 & 10.5312 & 3.7010 \\
\hline
\end{tabular}

${ }^{a}$ Calculation was carried out using $(R)$-epimers for $\mathbf{1 1}$ and 12. 
10. Human TK1 homology model docked with the $(R)$-epimer of $\mathbf{1 1}$

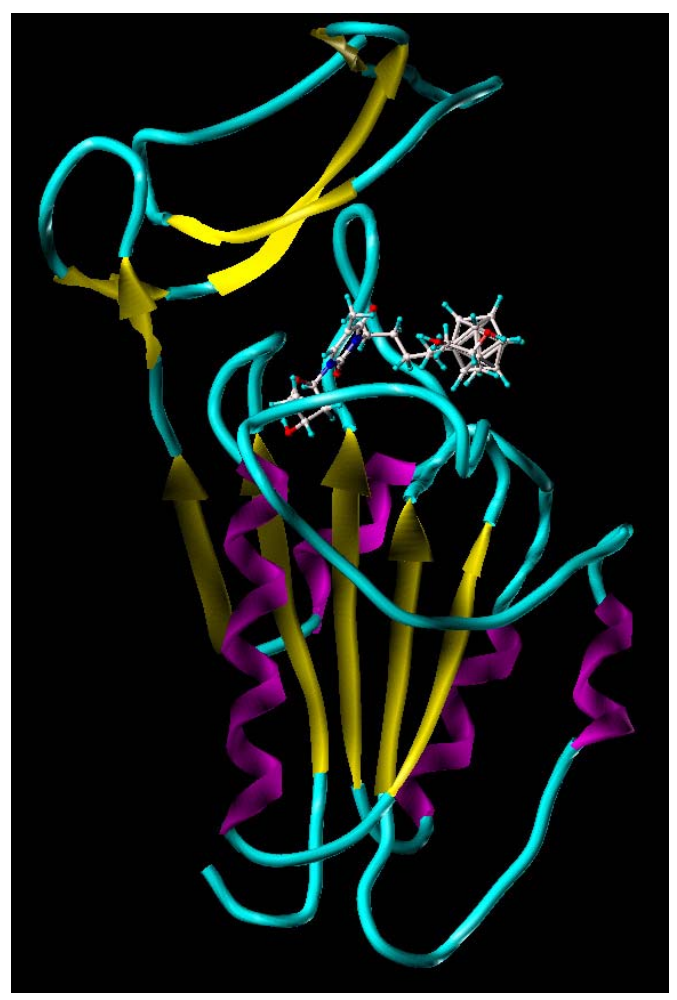

